Network Working Group

Request for Comments: 1592

Obsoletes: 1228

Category: Experimental
B. Wijnen

G. Carpenter

T.J. Watson Research Center, IBM Corp.

K. Curran

A. Sehgal

G. Waters

Bell Northern Research, Ltd.

March 1994

\title{
Simple Network Management Protocol \\ Distributed Protocol Interface \\ Version 2.0
}

Status of this Memo

This memo defines an Experimental Protocol for the Internet community. This memo does not specify an Internet standard of any kind. Discussion and suggestions for improvement are requested. Distribution of this memo is unlimited.

Table of Contents

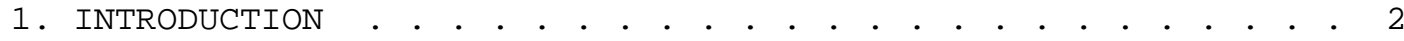

1.1 Motivation . . . . . . . . . . . . . . . . . . . . 3

1.2 Summary of Changes . . . . . . . . . . . . . . . . . . . . 4

2. THEORY OF OPERATION . . . . . . . . . . . . . . . . . . . . . . 5

2.1 Connection Establishment and Termination . • . . . . . . . 5

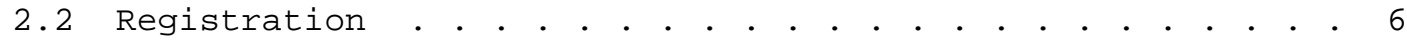

2.3 Normal Operation . . . . . . . . . . . . . . . . 6

2.4 DPI Architecture . . . . . . . . . . . . . . . . . . 6

3. SNMP DPI PROTOCOL . . . . . . . . . . . . . . . . . . . 110

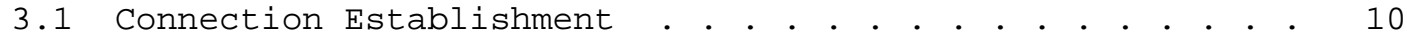

3.1.1 SNMP PDU to GET the Agent's DPI port . . . . . . . . . 11

3.1.2 SNMP PDU Containing the RESPONSE to the GET . . . . . 13

3.2 SNMP DPI Packet Formats . . • . . . . . . . . . . . 15

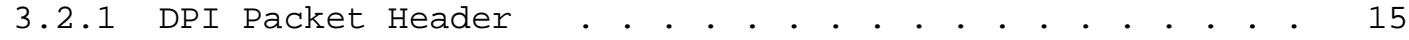

3.2.2 OPEN • • . . . . . . . . . . . . . . . . . . . . . . . 16

3.2.3 CLOSE . . . . . . . . . . . . . . . . . . . . . . . . . 18

3.2.4 ARE_YOU_THERE . . . . . . . . . . . . . . . . . . . . 19

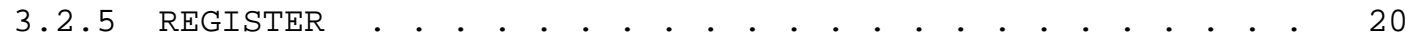

3.2.6 UNREGISTER • . . . . . . . . . . . . . . . . . . . . . . 22

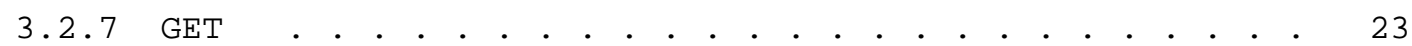

3.2.8 GETNEXT . . . . . . . . . . . . . . . . . . . . . 24

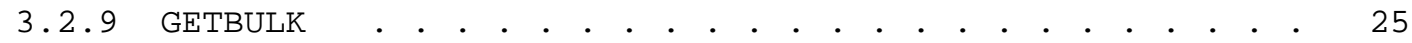

3.2.10 SET, COMMIT and UNDO . . . . . . . . . . . . . . . 26

3.2.11 RESPONSE . . . . . . . . . . . . . . . . . . . . . . 29

3.2.12 TRAP •. . . . . . . . . . . . . . . . . . . . . . . . 31

3.3 Constants and Values . . . . . . . . . . . . . . . 33 
3.3.1 Protocol Version and Release Values . . . . . . . . . 33

3.3.2 Packet Type Values . . . . . . . . . . . . . . . . . 34

3.3.3 Variable Type Values . . . . . . . . . . . . . . . . 35

3.3.4 Value Representation . . . . . . . . . . . . . . . 36

3.3.5 Character set selection . . . . . . . . . . . . . . . 36

3.3.6 Error Code Values for SNMP DPI RESPONSE packets . . . 37

3.3.7 UNREgISTER Reason Codes . . . . . . . . . . . . . . . 40

3.3.8 CLOSE Reason Codes . . . . . . . . . . . . . . . . . . 41

4. DPI 2.0 MIB DEFINITION . . . . . . . . . . . . . . . . . . . . . 41

5. SUBAGENT CONSIDERATIONS . . . . . . . . . . . . . . . . . . . . 42

5.1 DPI API . . . . . . . . . . . . . . . . . . . . . . . . 43

5.2 Overview of Request Processing . . . . . . . . . . . . . 44

5.2 .1 GET Processing . . . . . . . . . . . . . . . . . . . . 44

5.2.2 SET Processing . . . . . . . . . . . . . . . . . . . . 44

5.2.3 GETNEXT Processing . . . . . . . . . . . . . . . . . . 46

5.2.4 GETBULK Processing . . . . . . . . . . . . . . . . . 47

5.2 .5 OPEN Request . . . . . . . . . . . . . . . . . . . . 48

5.2.6 CLOSE Request . . . . . . . . . . . . . . . . . 49

5.2 .7 REGISTER Request . . . . . . . . . . . . . . . . . . . . 49

5.2.8 UNREGISTER Request . . . . . . . . . . . . . . . . . . 50

5.2 .9 TRAP Request . . . . . . . . . . . . . . . . . . . 51

5.2.10 ARE_YOU_THERE request . . . . . . . . . . . . . . . . 51

5.2.11 How to query the DPI port. . . . . . . . . . . . . . 51

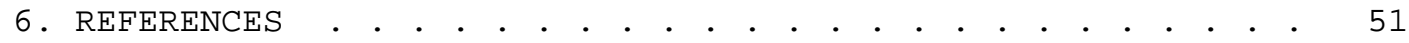

7. SECURITY CONSIDERATIONS • • • • • • • • • • • • • • • • 52

8. AUTHORS' ADDRESSES . . . . . . . . . . . . . . . . . . . . 53

9. SAMPLE SOURCES FOR ANONYMOUS FTP . . . . . . . . . . . . . . . $\quad . \quad 54$

\section{INTRODUCTION}

This RFC describes version 2.0 of a protocol that International Business Machines Corporation (IBM) has been implementing in most of its SNMP agents to allow dynamic extension of supported MIBs. Bell Northern Research (BNR) has also implemented a version of this protocol in some of its SNMP agents for the same reason.

The Simple Network Management Protocol (SNMP [1]) Distributed Protocol Interface (DPI) is an extension to SNMP agents that permits end-users to dynamically add, delete or replace management variables in the local Management Information Base without requiring recompilation of the SNMP agent. This is achieved by writing a socalled sub-agent that communicates with the agent via the SNMP-DPI.

For the author of a sub-agent, the SNMP-DPI eliminates the need to know the details of ASN.1 [2] or SNMP PDU (Protocol Data Unit) encoding/decoding $[1,3]$.

Versions 1.0 and 1.1 of this protocol have been in use within IBM 
since 1989 and is included in the SNMP agents for VM, MVS and OS/2. Version 1.2 of this protocol has been in use within BNR since 1992.

\subsection{MOTIVATION}

The Simple Network Management Protocol [1] defines a protocol that permits operations on a collection of variables. This set of variables is called the Management Information Base (MIB) and a core set of variables has previously been defined [4, 5]; however, the design of the MIB makes provision for extension of this core set. Thus, an enterprise or individual can define variables of their own which represent information of use to them. An example of a potentially interesting variable which is not in the core MIB would be CPU utilization (percent busy). Unfortunately, conventional SNMP agent implementations provide no means for an end-user to make available new variables.

Besides this, today there are many MIBs that people want to implement on a system. Without a capability for sub-agents, this requires all the MIBs to be implemented in one big monolithic agent, which is in many cases undesirable.

The SNMP DPI addresses these issues by providing a light-weight mechanism by which a process can register the existence of a MIB variable or a MIB sub-tree with the SNMP agent. Requests for the variable(s) that are received by the SNMP agent are passed to the process acting as a sub-agent. The sub-agent then returns an appropriate answer to the SNMP agent. The SNMP agent eventually packages an SNMP response packet and sends the answer back to the remote network management station that initiated the request.

Remote network management stations have no knowledge that the SNMP agent calls on other processes to obtain an answer. As far as they can tell, there is only one network management application (agent) running on the host.

At the San Diego IETF (March 1992) a BOF was held on multiplexing SNMP agent's requirements. Both the SMUX [6] and DPI [7] protocols were discussed, as well as other unpublished approaches. There was also discussion regarding a need for a standard for multiplexing SNMP agents or sub-agent support. At the end of the BOF, however, there was not enough support for defining a standard. This was due, at least partially, to a few well known SNMP authors who stated that the proxy and party support for SNMPv2 (SMP at the time) would solve the problem. 
Nevertheless, questions continue to be raised about sub-agent support (both in SNMP and SNMP2 mail lists) in spite of both SNMPV2 [8] being on the standard's track and SMUX being changed to a historic RFC. Furthermore, within IBM and BNR we continue to see a substantial and expanding use of the DPI protocol. with positive results.

Therefore, we believe that there is a place for a sub-agent protocol and we again offer this new version as an experimental protocol. We encourage people to try it and send us feedback. Depending on that feedback, we may decide to try to get onto the standards track at a later time.

During discussions about sub-agent interfaces at the San Diego BOF it also became clear that we should reduce the focus on the API for the sub-agent programmers. This RFC, therefore, specifies only the protocol to distribute SNMP requests from the main SNMP agent to the sub-agents. Programmers can build one or more Programming APIs on top of that protocol as needed, and sample API code is available from the authors of this document.

\subsection{SUMMARY OF CHANGES}

The following changes have been made since the initial definition of SNMP-DPI [7]. Some of these resulted from comparing the SMUX [6] and DPI [7] protocols.

- Documentation changes to cleanup and be more specific in some areas. Among other things, this includes:

- Defining that integers are in network byte order

- Defining the character set used for strings

- Defining how Displaystrings are handled.

- Including DPI20 MIB definition.

o Removal of the Programming API from the document.

o Addition of new DPI packet types:

- SNMP_DPI_OPEN for a sub-agent to open a "connection" with the DPI SNMP capable agent. The sub-agent must now identify itself and optionally provide a "password" for the connection.

- SNMP_DPI_CLOSE for the agent or sub-agent to close the connection in a graceful way.

- SNMP_DPI_ARE_YOU_THERE for the sub-agent to verify that the agent still knows about the sub-agent.

- SNMP_DPI_UNREGISTER for the agent or sub-agent to terminate the registration of a MIB variable or MIB sub-tree. 
- SNMP_DPI_COMMIT which instructs the sub-agent to actually commit a previous SNMP_DPI_SET request. This, together with the UNDO, allows DPI sub-agents to be compliant with SNMP in the sense that we can now handle the "as if simultaneous" requirement.

- SNMP_DPI_UNDO which instructs the sub-agent to UNDO a SET or COMMIT if such is needed.

- Multiple varBinds can now be exchanged in one DPI packet (for GET, GETNEXT, SET, TRAP). The sub-agent can specify the maximum it wants to handle per packet.

- The packet headers now contain a packet-ID (similar to SNMP request ID in SNMP PDU). This allows to match RESPONSE packets to REQUESTS, which is important for UDP based DPI-connections.

- The SNMP_DPI_REGISTER packet has new fields for time_out and for requested priority.

- The SNMP_DPI_TRAP packet allows to specify an enterprise OID. In addition, the generic and specific trap types are now 4 octets, so that we can pass the types correctly. - In general, the packets have a more consistent layout.

o The agent now sends a RESPONSE to a REGISTER request

- Addition of SNMPv2 error codes and value types.

\section{THEORY OF OPERATION}

\subsection{CONNECTION ESTABLISHMENT AND TERMINATION}

Communication between the SNMP Agent and its clients (sub-agents) takes place via a communication mechanism. The communication type can be either a logical stream connection (via TCP, for instance) or an unreliable datagram connection (UDP, for instance). It should be noted that other stream oriented transport communication mechanisms can also be used. For example, the VM SNMP agent allows DPI connections over IUCV (Inter-User Communications Vehicle) [9, 10]. Other than the connection establishment procedure, the protocol used is identical in these environments.

In Unix the number of processes is limited by the number of filedescriptors that can be opened. Since each TCP socket represents a file-descriptor, restricting SNMP-DPI protocol to TCP only connections would limit the number of sub-agents an agent could support. As a result, the some SNMP-DPI agents support both TCP and UDP socket type communication mechanisms for the SNMP-DPI protocol. 
Please note that in the following portion of this text the SNMP-DPI agent is referred simply as the agent.

Once the transport connection has been set up, the sub-agent must also initialize the logical connection with the agent. To do so it issues an OPEN request to the agent in which the sub-agent uniquely identifies itself and passes some other parameters to the agent, such as, the maximum number of varBinds per interaction it is prepared to handle, and the timeout the agent should use when waiting for a response from the sub-agent.

When the sub-agent prepares to stop or cease operations, it first issues a CLOSE to shut down the logical connection with the agent, and then closes the transport connection.

\subsection{REGISTRATION}

A sub-agent supports a collection of MIB variables or object identifiers (object IDs) that constitute its MIB (sub)tree. Each of these object IDs consists of a group ID and an instance ID. The group ID is the root of the sub-agent's MIB tree that it supports and the point of registration to the agent's MIB tree. The instance ID is the piece of the object Identifier that follows the group ID (registration point), so it is not an instance in the terms of the SNMP definition of an instance.

Regardless of the transport mechanism used, after establishing a connection to the agent, the sub-agent registers a branch (group ID) to the Agent's MIB tree. With the registration request, the subagent passes some parameters, such as, requested priority and a timeout value for this specific sub-tree.

The agent sends back a response to indicate success or failure of the registration request.

\subsection{NORMAL OPERATION}

Once the sub-agent has set up both the physical and logical connection to the agent, and once it has successfully registered the sub-tree(s) of the MIB(s) that it supports, it waits for requests from the SNMP agent or generates traps as required.

\section{4 DPI ARCHITECTURE}

These are the requests that can be initiated by the SNMP agent: GET, GETNEXT, GETBULK, SET, COMMIT, UNDO, UNREGISTER, and CLOSE. 
The first four of these correspond directly to SNMP requests that a network management station can make (By default a GETBULK request will be translated into multiple GETNEXT requests by the agent, but a sub-agent may request that the GETBULK be passed to it). The COMMIT, UNDO, UNREGISTER, ARE_YOU_THERE and CLOSE requests are specific SNMP-DPI requests. The sub-agent normally responds to a request with a RESPONSE packet. The CLOSE request is an exception for which the sub-agent only closes the physical connection.

These are the requests that can be initiated by a sub-agent:

OPEN, REGISTER, TRAP, UNREGISTER, ARE_YOU_THERE and CLOSE.

The agent responds to OPEN, REGISTER, UNREGISTER and ARE_YOU_THERE with a RESPONSE packet. The TRAP packet is just accepted and forwarded by the agent without returning any information to the subagent. The CLOSE packet is also just accepted by the agent upon which it closes the physical connection.

See Figure 1 for an overview of the DPI packet flow. 


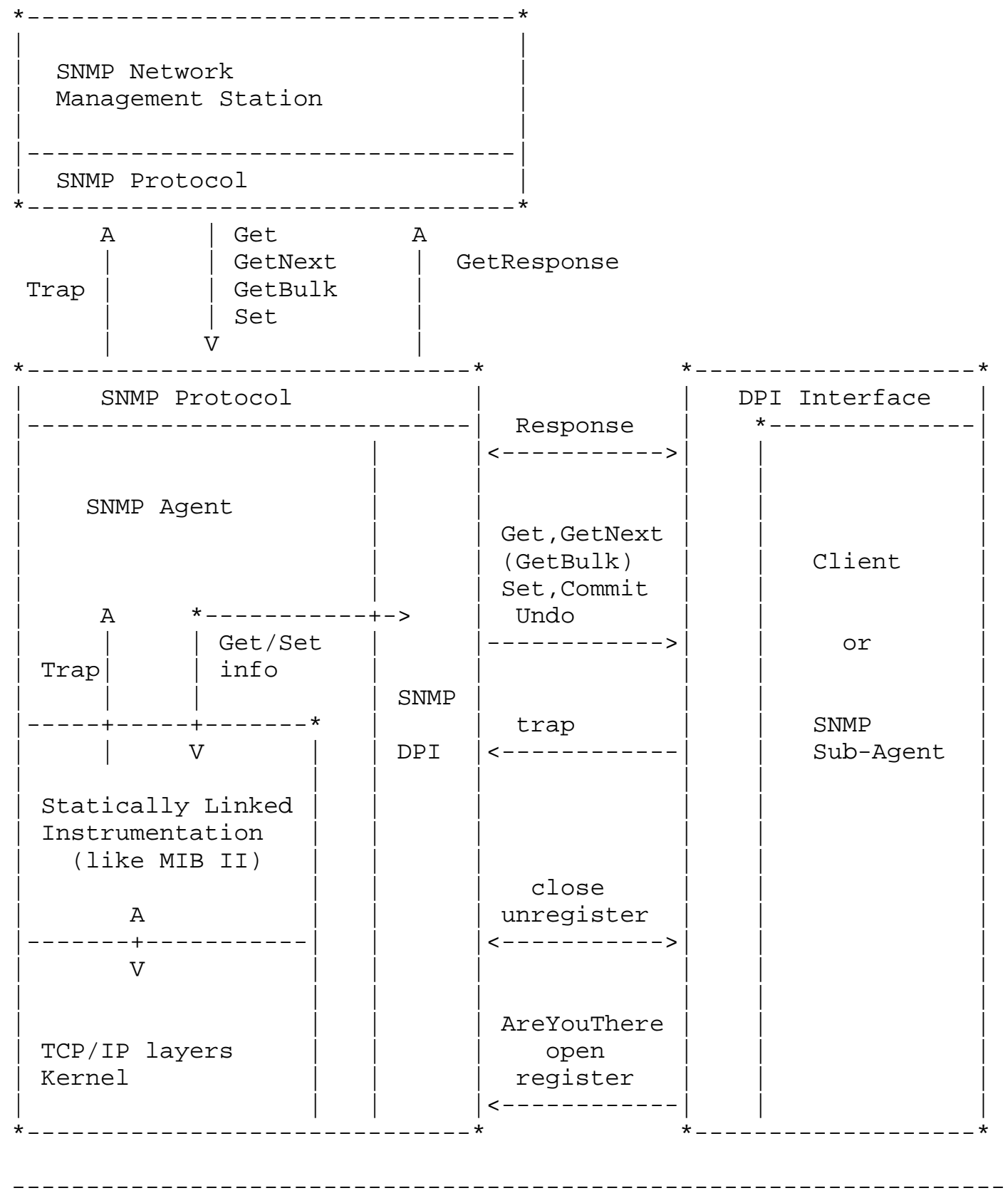

Figure 1. SNMP DPI overview 
Remarks for Figure 1:

- The SNMP agent communicates with the SNMP manager via the standard SNMP protocol.

o The SNMP agent communicates with some statically linked-in instrumentation (potentially for the MIB II), which in turn talks to the TCP/IP layers and kernel (operating system) in an implementation-dependent manner.

- An SNMP sub-agent, running as a separate process (potentially on another machine), can set up a connection with the agent. The sub-agent has an option to communicate with the SNMP agent through UDP or TCP sockets, or even through other mechanisms.

- Once the connection is established, the sub-agent issues a DPI OPEN and one or more REGISTER requests to register one or more MIB sub-trees with the SNMP agent.

- The SNMP agent responds to DPI OPEN and REGISTER requests with a RESPONSE packet, indicating success or failure.

- The SNMP agent will decode SNMP packets. If such a packet contains a Get or GetNext request for an object in a sub-tree registered by a sub-agent, it sends a corresponding DPI packet to the sub-agent. If the request is for a GetBulk, then the agent translates it into multiple DPI GETNEXT packets and sends those to the sub-agent. However, the sub-agent can request (in the REGISTER packet) that a GETBULK be passed to the sub-agent.

If the request is for a set, then the agent uses a 2-phase commit scheme and sends the sub-agent a sequence of SET/COMMIT, SET/UNDO or SET/COMMIT/UNDO DPI packets.

- The SNMP sub-agent sends responses back via a RESPONSE packet.

o The SNMP agent then encodes the reply into an SNMP packet and sends it back to the requesting SNMP manager.

- If the sub-agent wants to report an important state change, it sends a DPI TRAP packet to the SNMP agent which will encode it into an SNMP trap packet and send it to the manager(s).

o If the sub-agent wants to stop operations, it sends a DPI UNREGISTER and a DPI CLOSE packet to the agent. The agent sends a response to an UNREGISTER request.

- There is no RESPONSE to a CLOSE, the agent just closes the DPI connection. A CLOSE implies an UNREGISTER for all registrations that exist for the DPI connection being CLOSED.

- An agent can send DPI UNREGISTER (if a higher priority registration comes in or for other reasons) to the sub-agent, the sub-agent then responds with a DPI RESPONSE packet.

- An agent can also (for whatever reason) send a DPI CLOSE to indicate it is terminating the DPI connection.

- A sub-agent can send an ARE_YOU_THERE to verify that the "connection" is still open. If so, the agent sends a RESPONSE with no error, otherwise, it may send a RESPONSE with an error 
indication, or not react at all.

3. SNMP DPI PROTOCOL

This section describes the actual protocol used between the SNMP agent and sub-agents.

\subsection{CONNECTION ESTABLISHMENT}

In a TCP/IP environment, the SNMP agent listens on an arbitrary TCP/UDP port for a connection request from a sub-agent. It is important to realize that a well-known port is not used: every invocation of the SNMP agent will potentially result in a different TCP/UDP port being used.

A sub-agent needs to determine this port number to establish a connection. The sub-agent learns the port number from the agent by sending it one conventional SNMP get-request PDU. The port numbers are maintained by the SNMP agent as the objects whose identifiers are:
$1 \cdot 3 \cdot 6 \cdot 1 \cdot 4 \cdot 1 \cdot 2 \cdot 2 \cdot 1 \cdot 1 \cdot 0$
dpiport. 0
(old DPI $1 . x$ form)
1.3 .6 .1 .4 .1 .2 .2 .1 .1 .1 .0
dpiPortForTCP. 0
$1.3 \cdot 6 \cdot 1 \cdot 4 \cdot 1 \cdot 2 \cdot 2 \cdot 1 \cdot 1 \cdot 2 \cdot 0$
dpiportForUDP. 0

These variables are registered under the IBM enterprise-specific tree. See 4, "DPI 2.0 MIB definition" for more information. The SNMP agent replies with a conventional SNMP response PDU that contains the port number to be used. This response is examined by the sub-agent and the port number is extracted. The sub-agent then establishes the connection to the specified port.

On the surface, this procedure appears to mean that the sub-agent must be able to create and parse SNMP packets, but this is not the case. A DPI Application Programming Interface (API) normally provides a library routine, query_DPI_port(), which can be used to generate and parse the required SNMP packets. This very small routine (under 100 lines of $\mathrm{C}$ ), does not greatly increase the size of any sub-agent.

NOTE: Since this RFC does not define an API, the actual code of and interface to a query_DPI_port() type of function depends on the implementation.

For completeness, byte-by-byte descriptions of the packets to be generated by an SNMP DPI API routine query_DPI_port() are provided below. This is probably of little interest to most readers and reading the source of a query_DPI_port() function provides much of 
the same information.

\subsubsection{SNMP PDU TO GET THE AGENT'S DPI PORT}

As noted, before a TCP/UDP connection to the SNMP agent can be made, the sub-agent must learn which port that the agent is listening on. To do so, it can issue an SNMP GET for the variable dpiPortForTCP.0 $(1.3 .6 .1 .4 .1 .2 .2 .1 .1 .1 .0)$ or variable dpiportForUDP.0 $(1.3 \cdot 6 \cdot 1 \cdot 4 \cdot 1 \cdot 2 \cdot 2 \cdot 1 \cdot 1 \cdot 2 \cdot 0)$.

The SNMP PDU can be constructed as shown below. This PDU must be sent to UDP port 161 on the host where the agent runs (probably the same host where the sub-agent runs).

The (SNMPV1) packet shown below is for the TCP port. 


\begin{tabular}{|c|c|c|}
\hline OFFSET & VALUE & FIELD \\
\hline 0 & $0 \times 30$ & ASN.1 header \\
\hline 1 & $37+$ len & PDU_length, see formula below \\
\hline 2 & $0 \times 020 \times 010 \times 00$ & $\begin{array}{l}\text { SNMP version: } \\
\text { (integer, length }=1, \text { value }=0 \text { ) }\end{array}$ \\
\hline 5 & $0 \times 04$ & community name (string) \\
\hline 6 & len & length of community name \\
\hline 7 & community name & varies \\
\hline $7+$ len & $0 \times a 00 \times 1 c$ & $\begin{array}{l}\text { SNMP GET request: } \\
\text { request_type }=0 \times a 0,1 \text { engt } h=0 \times 1 \mathrm{C}\end{array}$ \\
\hline $7+1 e n+2$ & $0 \times 02 \quad 0 \times 01 \quad 0 \times 01$ & $\begin{array}{l}\text { SNMP request ID: } \\
\text { integer, length }=1, I D=1\end{array}$ \\
\hline $7+1 e n+5$ & $0 \times 02 \quad 0 \times 01 \quad 0 \times 00$ & $\begin{array}{l}\text { SNMP error status: } \\
\text { integer, length }=1 \text {, error }=0\end{array}$ \\
\hline $7+1 e n+8$ & $0 \times 020 \times 010 \times 00$ & $\begin{array}{l}\text { SNMP index: } \\
\text { integer, length }=1, \text { index }=0\end{array}$ \\
\hline $7+1 e n+11$ & $0 \times 30 \quad 0 \times 11$ & varBind list, length $=0 \times 11$ \\
\hline $7+1 e n+13$ & $0 \times 300 \times 0 f$ & varBind, length $=0 \times 0 f$ \\
\hline $7+$ len +15 & $0 \times 060 \times 0 b$ & Object ID, length $=0 \times 0 b$ \\
\hline
\end{tabular}




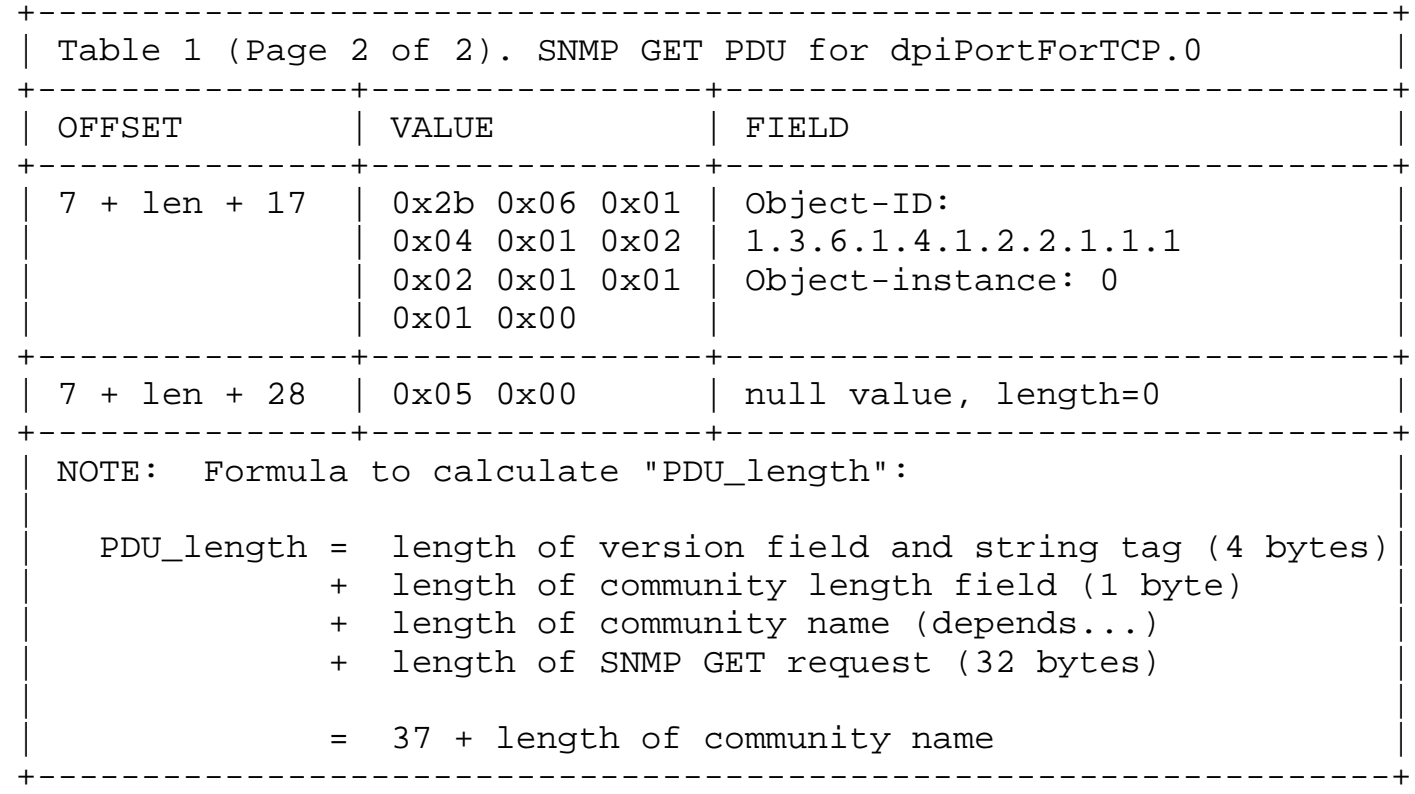

\subsubsection{SNMP PDU CONTAINING THE RESPONSE TO THE GET}

Assuming that no errors occurred, the port is returned in the last few octets of the received packet. In the simple case, where the port number will be between 1024 and 16,385, the format of the packet is shown below.

Note: In practice, the port number can be any positive number in the range from 1 through 65,535. A port number of 0 means that the agent does not have a dpiport defined for the requested protocol. So the actual port value maybe in the last 1,2 or 3 octets. The sample implementation code shows how to handle the response to cover all those cases, including error conditions.

Note: The (SNMPV1) packet shown below is for the TCP port.

\begin{tabular}{|c|c|c|}
\hline OFFSET & VALUE & FIELD \\
\hline 0 & $0 \times 30$ & ASN.1 header \\
\hline 1 & $39+1 e n$ & length, see formula below \\
\hline
\end{tabular}




\begin{tabular}{|c|c|c|}
\hline OFFSET & VALUE & FIELD \\
\hline 2 & $0 \times 020 \times 010 \times 00$ & $\begin{array}{l}\text { version } \\
\text { (integer, length }=1, \text { value }=0 \text { ) }\end{array}$ \\
\hline 5 & $0 \times 04$ & community name (string) \\
\hline 6 & len & length of community name \\
\hline 7 & community name & \\
\hline $7+1 e n$ & $0 x a 20 \times 1 e$ & $\begin{array}{l}\text { SNMP RESPONSE: } \\
\text { request_type }=0 \times a 2, \text { length }=0 \times 1 e\end{array}$ \\
\hline $7+1 e n+2$ & $0 \times 020 \times 010 \times 01$ & $\begin{array}{l}\text { SNMP request ID: } \\
\text { integer, length }=1, I D=1\end{array}$ \\
\hline $7+1 e n+5$ & $0 \times 020 \times 010 \times 00$ & $\begin{array}{l}\text { SNMP error status: } \\
\text { integer, length }=1 \text {, error }=0\end{array}$ \\
\hline $7+1 e n+8$ & $0 \times 020 \times 010 \times 00$ & $\begin{array}{l}\text { SNMP index: } \\
\text { integer, length }=1, \text { index }=0\end{array}$ \\
\hline $7+1 e n+11$ & $0 \times 30 \quad 0 \times 13$ & varBind list, length $=0 \times 13$ \\
\hline $7+1 e n+13$ & $0 \times 300 \times 11$ & varBind, length=0x11 \\
\hline $7+$ len +15 & $0 \times 060 \times 0 b$ & Object ID, length=0x0b \\
\hline $7+$ len +17 & $\begin{array}{lll}0 \times 2 b & 0 \times 06 & 0 \times 01 \\
0 \times 04 & 0 \times 01 & 0 \times 02 \\
0 \times 02 & 0 \times 01 & 0 \times 01 \\
0 \times 01 & 0 \times 00 & \end{array}$ & $\begin{array}{l}\text { Object-ID: } \\
1 \cdot 3 \cdot 6 \cdot 1 \cdot 4 \cdot 1 \cdot 2 \cdot 2 \cdot 1 \cdot 1 \cdot 1 \\
\text { object-instance: } 0\end{array}$ \\
\hline $7+1 e n+28$ & $0 \times 020 \times 02$ & integer, length $=2$ \\
\hline $7+1 e n+30$ & MSB LSB & port number (MSB, LSB) \\
\hline
\end{tabular}




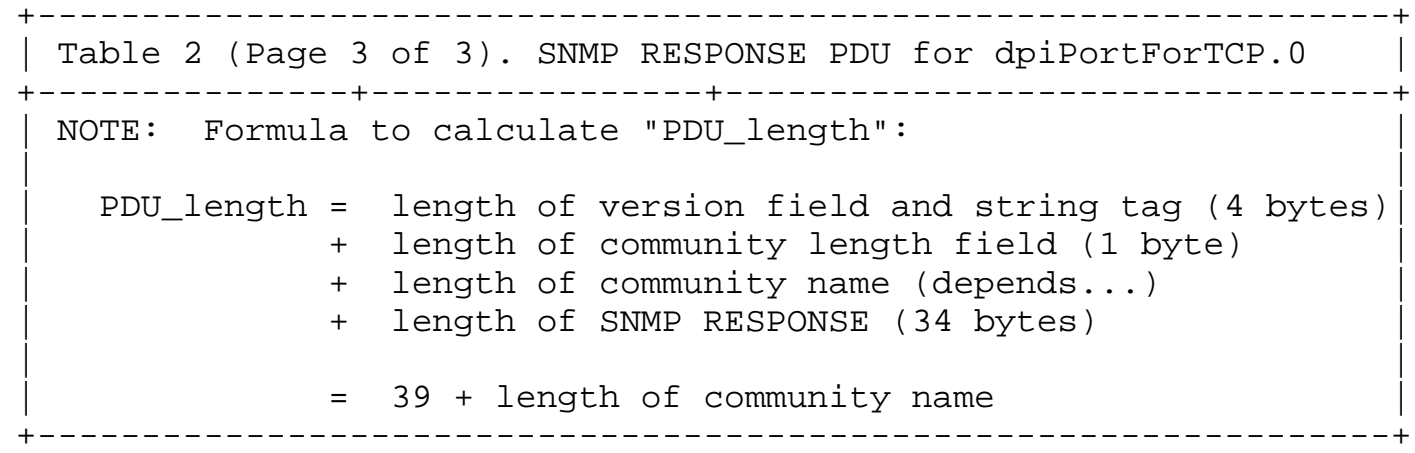

\subsection{SNMP DPI PACKET FORMATS}

Each request to, or response from, the agent or sub-agent is constructed as a "packet" and is written to the stream.

Each packet is prefaced with the length of the data remaining in the packet. The length is stored in network byte order, the most significant byte (MSB) first, least significant byte (LSB) last. If we consider a stream connection (like TCP), the receiving side will read the packet by doing something similar to:

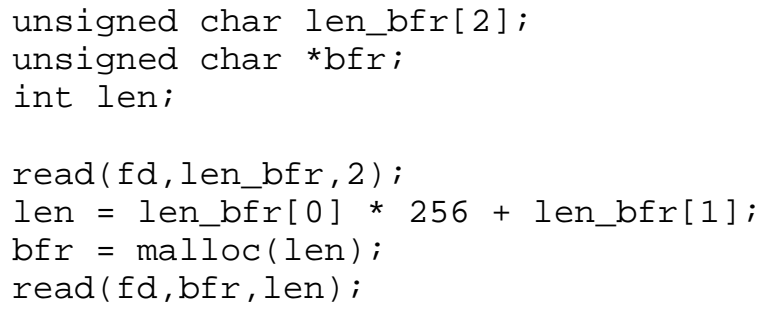

Note: The above example makes no provisions for error handling or a read returning less than the requested amount of data, and it is not intended to be used literally.

\subsubsection{DPI PACKET HEADER}

The first part of every packet identifies the application protocol being used as well as some version information. The protocol major version is intended to indicate, in broad terms, what version of the protocol is used. The protocol minor version is intended to identify major incompatible versions of the protocol. The protocol release is intended to indicate incremental modifications to the protocol. The constants that are valid for these fields are defined in Table 15. 
The next field, present in all packets, is the packet ID. It contains packet identification that can help an agent or sub-agent match responses with request. This is useful with UDP connections over which packets can be lost. The packet ID is a monotonically increasing unsigned 16-bit integer which wraps at its maximum value.

The next field, present in all packets, is the packet type. It indicates what kind of packet we're dealing with (OPEN, REGISTER, GET, GETNEXT, GETBULK, SET, COMMIT, UNDO, TRAP, RESPONSE, UNREGISTER, or CLOSE). The permitted values for this field are defined in Table 16.

\begin{tabular}{|c|c|}
\hline OFFSET & FIELD \\
\hline 0 & packet length to follow (MSB to LSB) \\
\hline 2 & protocol major version \\
\hline 3 & protocol minor version \\
\hline 4 & protocol release \\
\hline 5 & packet id (MSB to LSB) \\
\hline 7 & packet type \\
\hline
\end{tabular}

From this point onwards, the contents of the packet are defined by the protocol being used. The remainder of this section describes:

- Layout of packets for the SNMP DPI protocol, version 2.0 .

- Constants as defined with this version of the protocol.

\subsubsection{OPEN}

In order for a sub-agent to communicate with a DPI capable SNMP agent, it must first send an SNMP DPI OPEN request to the agent to setup the "connection" with that agent.

Such a packet contains the standard SNMP DPI header plus OPEN specific data. This data consists of: 
- a timeout value (in seconds).

This is a requested timeout value to be used for all requests for objects for which there is no timeout value specified for the sub-tree under which the object is registered. If you specify a zero timeout value, then the agent will use its own default timeout value. If you want a larger value than the default value, then you can specify it here. However, the agent may have a maximum value that you can never exceed. If you do ask for a larger timeout than that maximum, the agent will set it at the maximum it accepts.

o the maximum number of varBinds per DPI packet that the sub-agent is prepared to handle.

- Selected character set to be used for the representation of the OBJECT ID strings and Displaystrings.

The choices are the native character set (0) or the ASCII character set (1). See 3.3.5, "Character set selection" for more information in character set selection. An agent may choose to support only the native character set.

- null terminated sub-agent ID, which is a unique ASN.1 OBJECT identifier, so in dotted ASN.1 notation. This string is represented in the selected character set.

- null terminated sub-agent description, which is a Displaystring describing the sub-agent. This string is represented in the selected character set. This may be the null-string if there is no description.

o optionally a password that the agent uses to validate the sub-agent. It depends on the agent implementation if a password is required. If no password is passed, the length must be specified as zero.

The sub-agent must expect a response indicating success or failure. See Table 19 for the valid codes in a DPI RESPONSE to a DPI OPEN request.

If the error_code in the RESPONSE is not SNMP_ERROR_DPI_noError, then the agent closes the connection. 


\begin{tabular}{|c|c|}
\hline OFFSET & FIELD \\
\hline 0 & packet length to follow (MSB to LSB) \\
\hline 2 & protocol major version \\
\hline 3 & protocol minor version \\
\hline 4 & protocol release \\
\hline 5 & packet id (MSB to LSB) \\
\hline 7 & packet type $=$ SNMP_DPI_OPEN \\
\hline 8 & | requested overall timeout (seconds, MSB to LSB) \\
\hline 10 & max varBinds per DPI packet (MSB to LSB) \\
\hline 12 & | Selected character set (0=Native, $1=$ ASCII) \\
\hline & null terminated sub-agent ID (OID) \\
\hline $13+\mathrm{L} 1$ & | null terminated sub-agent Description \\
\hline $13+\mathrm{L} 2$ & | password length (zero if no password, MSB to LSB) \\
\hline $15+\mathrm{L} 2$ & | password (if any) \\
\hline \multicolumn{2}{|l|}{ NOTE : } \\
\hline \multicolumn{2}{|c|}{$0 \quad$ L1 $=$ strlen (sub-agent ID) +1} \\
\hline \multicolumn{2}{|c|}{$\circ \quad \mathrm{L} 2=\mathrm{L} 1+$ strlen (sub-agent Description) +1} \\
\hline \multicolumn{2}{|c|}{ o OID and Description strings use selected character set } \\
\hline
\end{tabular}

\subsubsection{CLOSE}

In order for a sub-agent to close the "connection" with the DPI capable SNMP agent, it must send an SNMP DPI CLOSE request to the agent. The agent will not send a response, but closes the physical connection and implicitly unregisters any sub-trees related to the connection.

An agent may also send to the sub-agent an SNMP DPI CLOSE packet that contains the standard SNMP DPI header plus CLOSE specific data. This 
data consists of:

- a reason code for closing. See Table 21 for a list of valid reason codes.

\begin{tabular}{|c|c|}
\hline OFFSET & FIELD \\
\hline 0 & packet length to follow (MSB to LSB) \\
\hline 2 & protocol major version \\
\hline 3 & protocol minor version \\
\hline 4 & protocol release \\
\hline 5 & packet id (MSB to LSB) \\
\hline 7 & packet type $=$ SNMP_DPI_CLOSE \\
\hline 8 & reason code (1 octet) \\
\hline
\end{tabular}

\subsubsection{ARE_YOU_THERE}

An ARE_YOU_THERE packet allows a sub-agent to determine if it still has a DPI connection with the agent. This packet is necessary because a sub-agent passively awaits requests from an agent and normally will not detect problems with an agent connection in a timely manner. (In contrast, an agent becomes aware of any sub-agent connection problem in a timely manner because it sets a timeout when sending request).

A sub-agent can send a SNMP DPI ARE_YOU_THERE packet to an agent which will then return a RESPONSE with a zero error code and a a zero error index if the connection is healthy. Otherwise, the agent may return a RESPONSE with an error indication. If the connection is broken, the sub-agent will see no response at all.

An ARE_YOU_THERE packet contains the standard SNMP DPI header with no additional data. 


\begin{tabular}{|c|c|}
\hline OFFSET & FIELD \\
\hline 0 & packet length to follow (MSB to LSB) \\
\hline 2 & protocol major version \\
\hline 3 & protocol minor version \\
\hline 4 & protocol release \\
\hline 5 & packet id (MSB to LSB) \\
\hline 7 & packet type $=$ SNMP_DPI_ARE_YOU_THERE \\
\hline
\end{tabular}

\subsubsection{REGISTER}

In order to register a branch in the MIB tree, an SNMP sub-agent sends an SNMP DPI REGISTER packet to the agent.

Such a packet contains the standard SNMP DPI header plus REGISTER specific data. This data consists of:

- a requested priority.

There are 2 special values, namely minus one (-1, requests best available priority) and zero (0, requests next better priority than the highest priority in use). Any other value requests a specific priority or the next best priority if already in use). The lower the number, the better the priority. An agent will send requests to only the one sub-agent that has registered with the best priority. The agent returns the actual priority assigned in the RESPONSE packet in the error_index field.

- a requested timeout.

If a zero value is specified, then the agent uses the timeout value specified in the DPI OPEN request.

If you want a shorter or longer timeout value for this specific sub-tree, then you specify it here. The agent has a maximum timeout it will allow in this field. The agent will use this value (or its maximum) to await a response to requests for this sub-tree.

- an indication as to whether the sub-agent wishes to handle MIB view selection (SNMPV1 community string authentication) in subsequent GET, GETNEXT or SET, COMMIT, UNDO requests. Not all DPI capable agents need to support this feature, but they must at least recognize this indication and give an appropriate 
response if they do not support it.

- an indication as to whether the sub-agent wishes to handle the GETBULK itself. If not, then the agent will translate a GETBULK into multiple GETNEXT requests.

Not all DPI capable agents need to support this feature. They may opt to always translate a GETBULK into multiple GETNEXT requests. In this case the agent will send the appropriate RESPONSE to indicate this.

- the group ID (sub-tree) to be registered (with trailing dot). The group ID is represented in the selected character set as specified in DPI OPEN packet.

The agent will respond with an SNMP DPI RESPONSE packet indicating registration error or success. The packet ID of the response will be the same as that for the REGISTER request to which this is a response.

The group ID will be the same as that specified in the REGISTER request. There will be no instance returned (e.g. NULL string for instance ID). The value will be an SNMP_TYPE_NULL value with a zero length. 


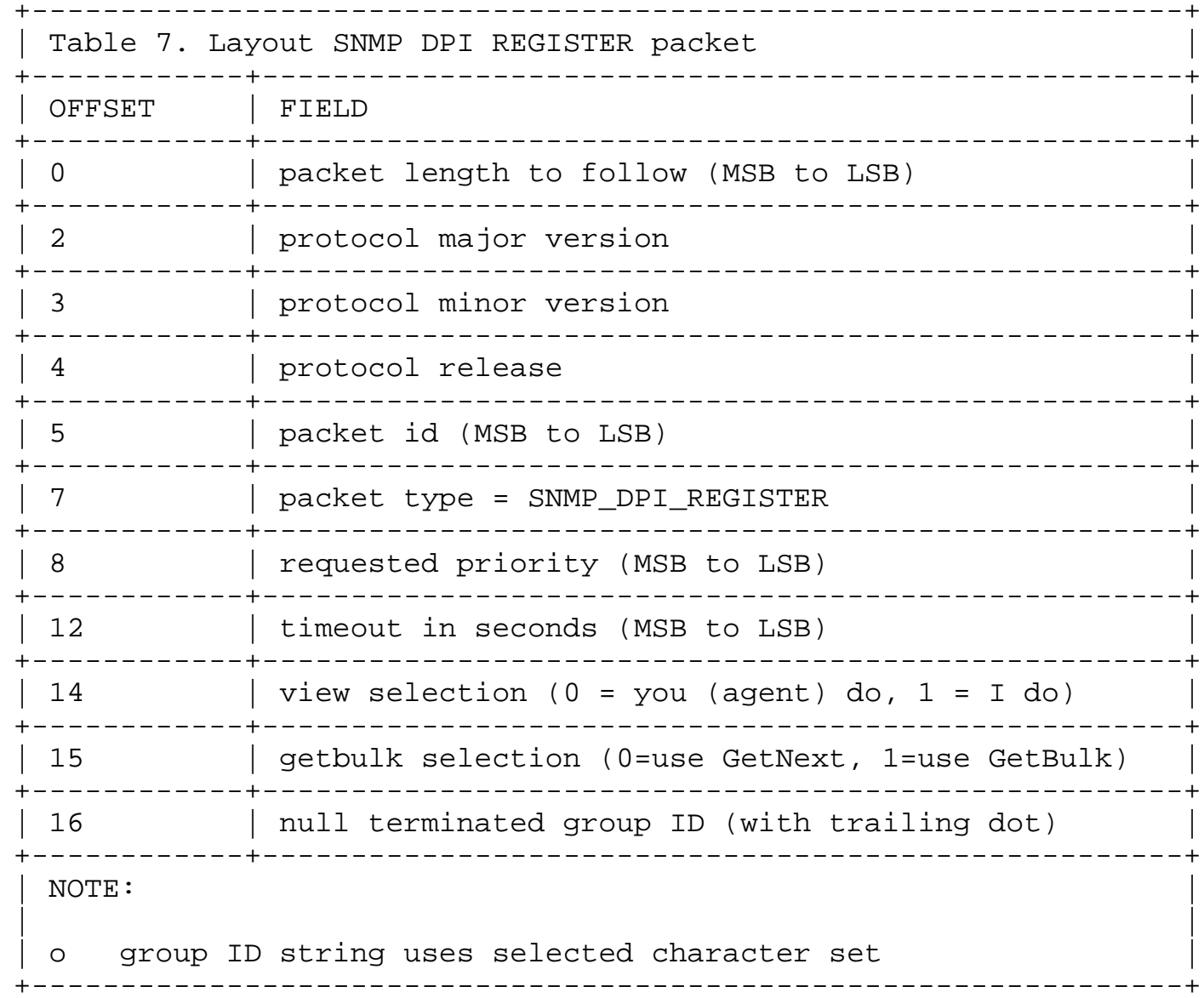

\subsubsection{UNREGISTER}

In order to unregister a branch in the MIB tree, an SNMP sub-agent sends an SNMP DPI UNREGISTER packet to the agent.

Such a packet contains the standard SNMP DPI header plus UNREGISTER specific data: a null terminated string (represented in the selected character set) representing the group ID in ASN.1 dotted notation and an indication as to the reason for the unregister (see table 14).

The agent will respond with an SNMP DPI RESPONSE packet indicating error or success. The packet ID of the response will be the same as that for the UNREGISTER request to which this is a response.

The group ID will be the same as that specified in the UNREGISTER request. There will be no instance returned (e.g. NULL string for 
instance ID). The value will be an SNMP_TYPE_NULL value with a zero length.

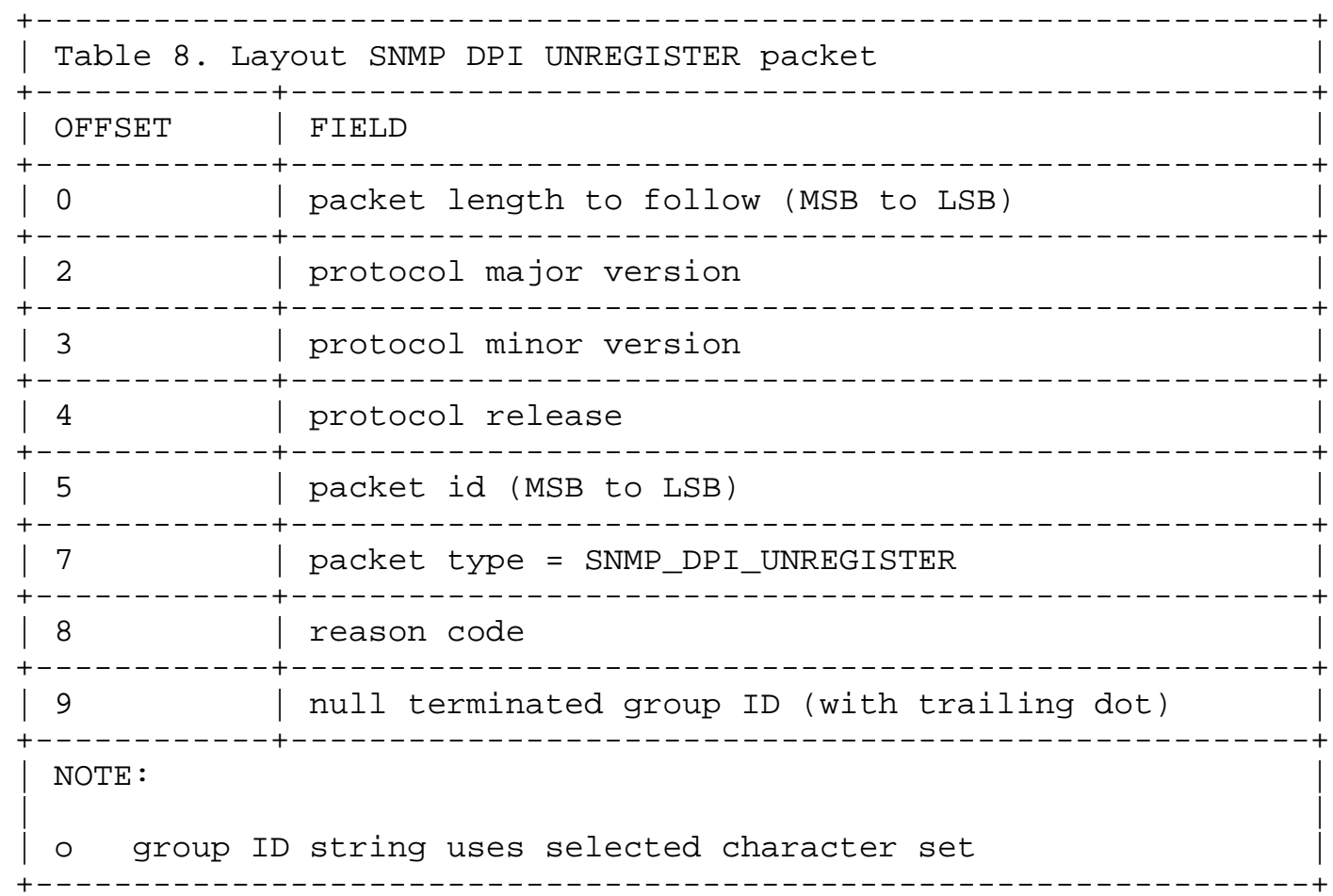

\subsubsection{GET}

When the SNMP agent receives a PDU containing an SNMP GET request for a variable that resides in a sub-tree registered by a sub-agent, it passes an SNMP DPI GET packet to the sub-agent.

Such a packet contains the standard SNMP DPI header plus GET specific data:

- the community name used in the SNMP PDU. The length is zero unless view handling was selected by the sub-agent. The length is also zero if the SNMP PDU was not in SNMPv1 format.

- per varBind two null terminated strings (in the selected character set) representing the group and instance ID in ASN.1 dotted notation. 


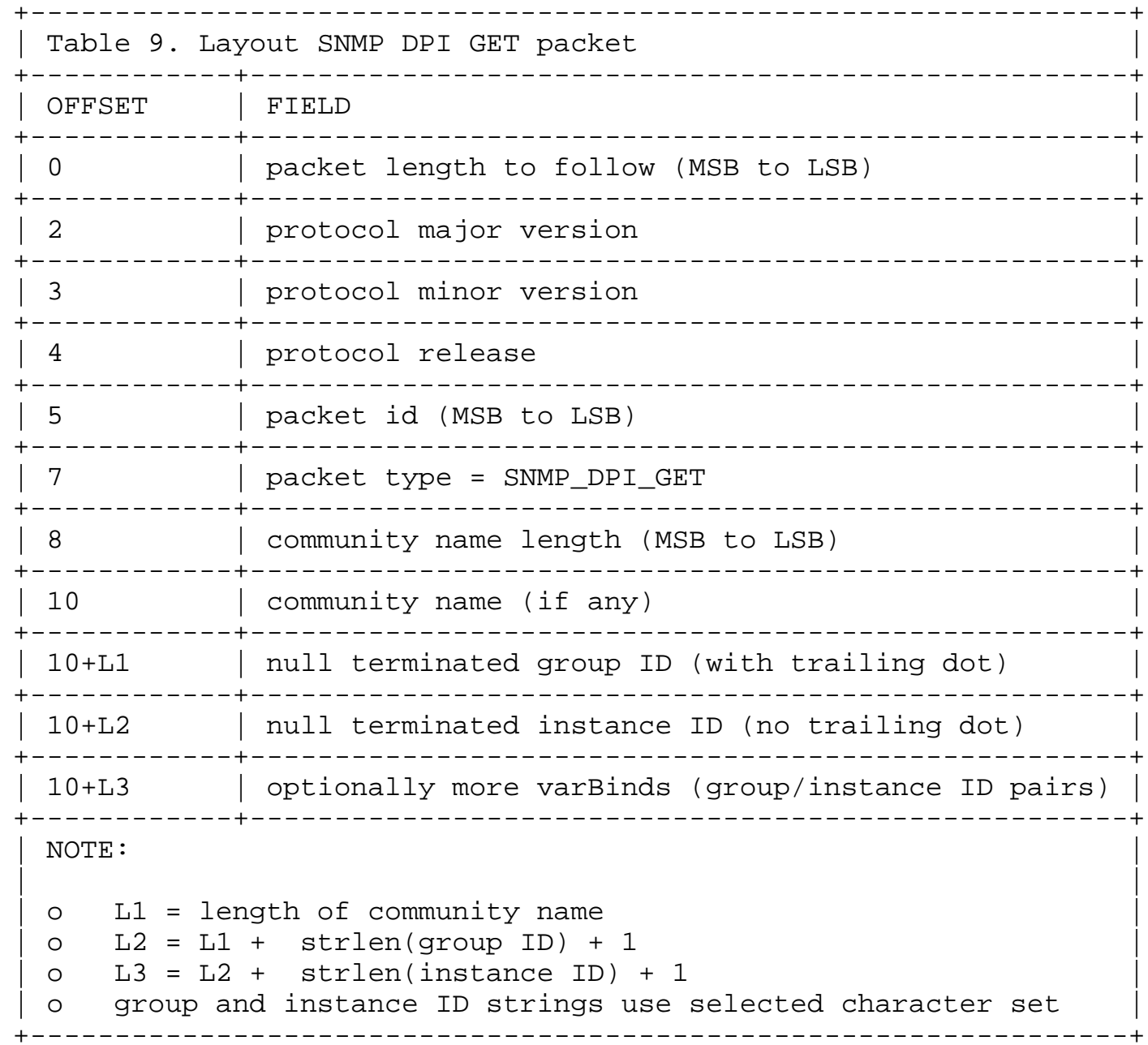

\subsubsection{GETNEXT}

When the SNMP agent receives a PDU containing an SNMP GETNEXT request for a variable for which a sub-agent may be authoritative, it passes an SNMP DPI GETNEXT packet to the sub-agent.

Such a packet contains the standard SNMP DPI header plus GETNEXT specific data:

- the community name used in the SNMP PDU. The length is zero unless view handling was selected by the sub-agent. The length is also zero if the SNMP PDU was not in SNMPv1 format.

- per varBind two null terminated strings (in the selected 
character set) representing the group and instance ID in ASN.1 dotted notation.

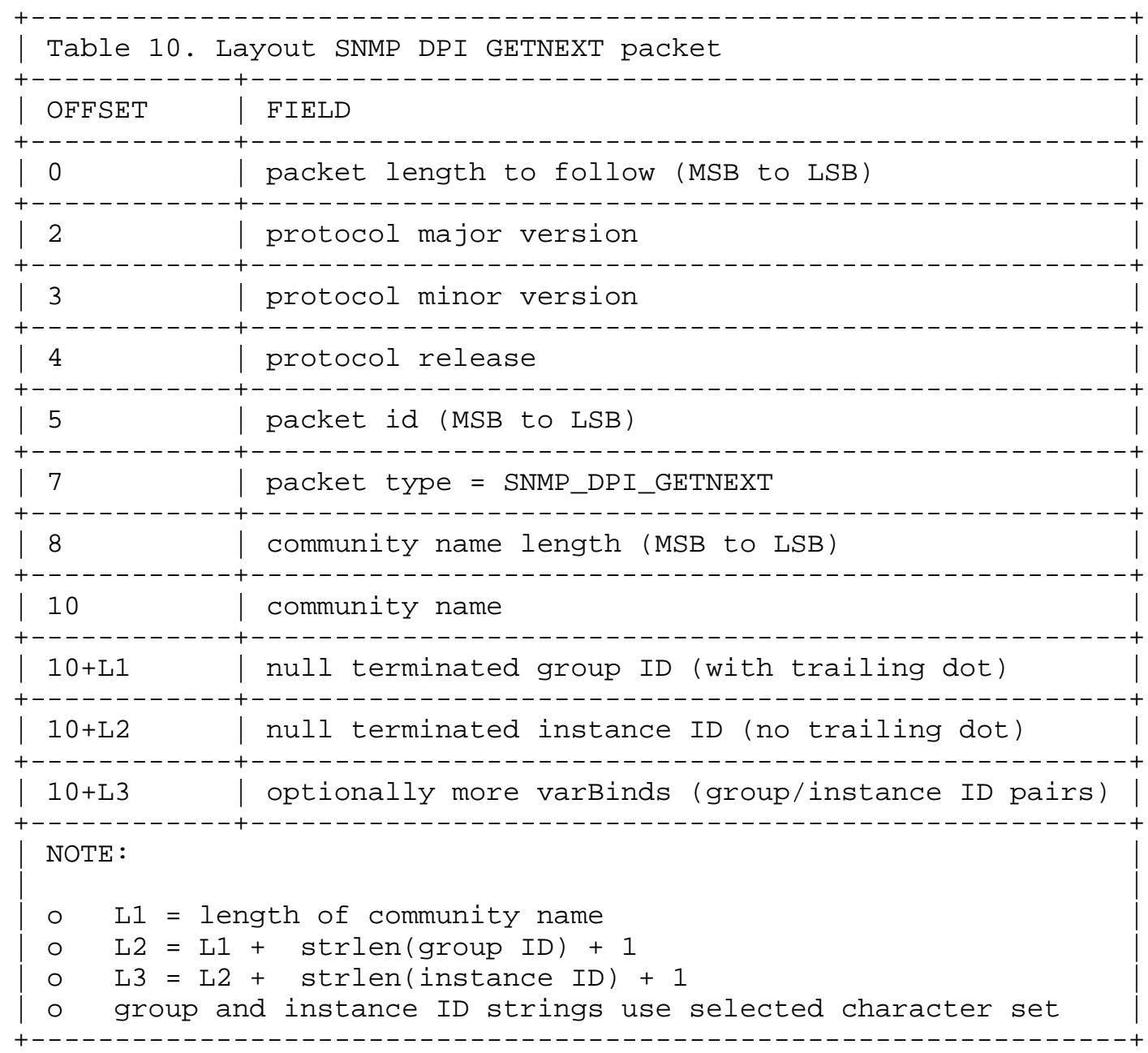

\subsubsection{GETBULK}

When the SNMP agent receives a PDU containing an SNMP GETBULK request that includes variables for which a sub-agent may be authoritative, it checks if the sub-agent wants to handle the GETBULK itself (as specified at registration time). If so, it sends an SNMP DPI GETBULK packet to the sub-agent.

Such a packet contains the standard SNMP DPI header plus GETBULK specific data: 


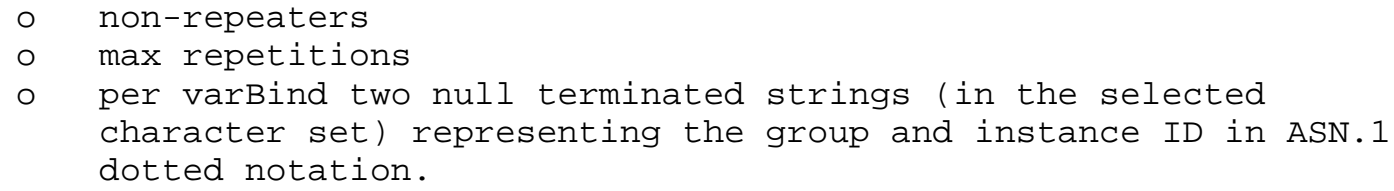

\subsubsection{SET, COMMIT AND UNDO}

When the SNMP agent receives a PDU containing an SNMP SET request for a variable that is in a sub-tree registered by a sub-agent, it passes one of 3 sequences of SNMP DPI packets to the sub-agent: 
SET, COMMIT

This is the normal sequence. The SET request is the first phase. The sub-agent must verify that the SET request is valid and that the resources needed are available. The COMMIT request comes next. The sub-agent must now effectuate the SET request.

O SET, UNDO

If an SNMP packet has a SET request for multiple varBinds that reside in different sub-trees, then the agent first sends a SET to all sub-agents. If any sub-agent returns an error on the SET, then the agent sends UNDO to those sub-agents that returned no error on the SET, meaning the SET is being canceled.

o SET, COMMIT, UNDO

In the very rare circumstance where all sub-agents have responded error-free to a SET and where one of them fails to perform the COMMIT, then the agent sends an UNDO to all involved sub-agents (also those who completed COMMIT). Sub-agents should try, to the best of their ability, to never let a commit fail and to undo an already committed set if asked to do so.

Such packets contain the standard SNMP DPI header plus SET specific data:

- the community name used in the SNMP PDU. The length is zero unless view handling was selected by the sub-agent. The length is also zero if the SNMP PDU was not in SNMPV1 format.

$\circ$ per varBind:

- two null terminated strings (in the selected character set) representing the group and instance ID in ASN.1 dotted notation.

- the type, value length and value to be set.

The permitted types for the type field are defined in Table 17. See 3.3.4, "Value Representation" for information on how the value data is represented in the packet value field. 


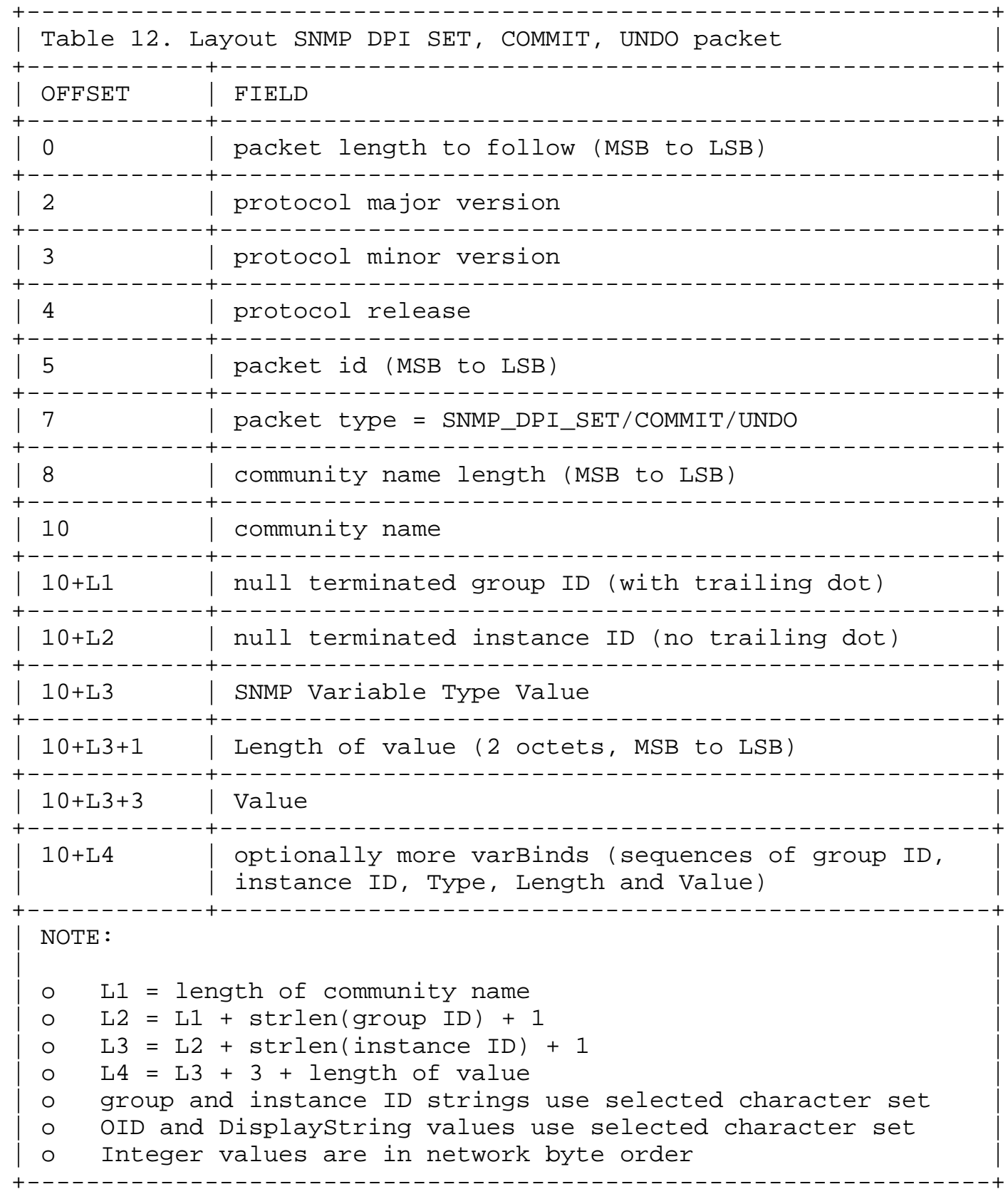




\subsubsection{RESPONSE}

An SNMP sub-agent must respond to a GET, GETNEXT, GETBULK, SET, COMMIT, UNDO or UNREGISTER request that it has received from the agent (unless it fails or has a bug $;^{-)}$)). To do so, it sends an SNMP DPI RESPONSE packet to the agent.

Such a packet contains the standard SNMP DPI header plus RESPONSE specific data:

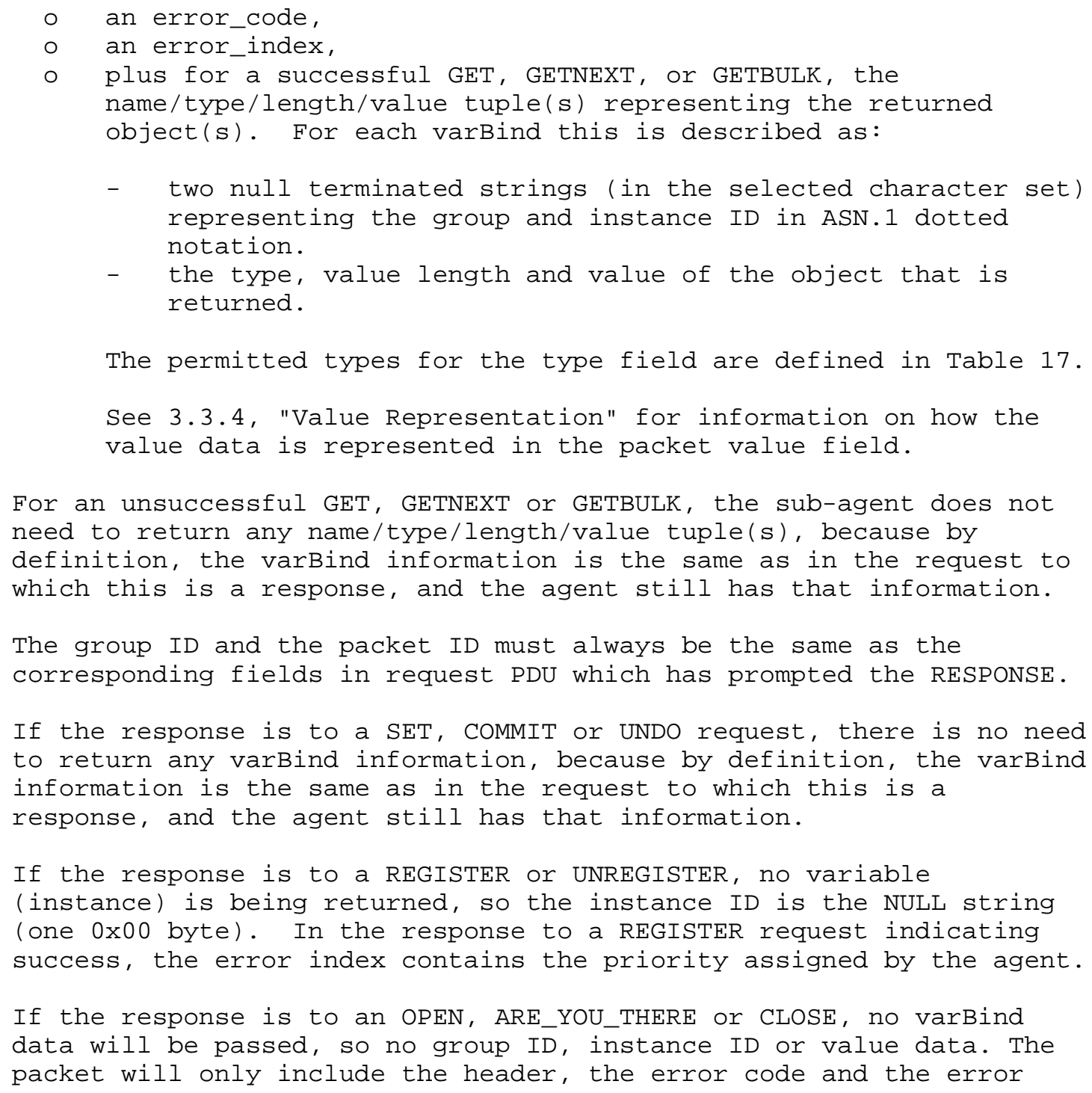




\section{2 .12 TRAP}

An SNMP sub-agent can request the agent to generate an SNMPv1 or SNMPV2 TRAP (depending on the trap destinations defined at the agent) by sending an SNMP DPI TRAP packet to the agent.

Such a packet contains the standard SNMP DPI header plus TRAP specific data:

- the generic and specific trap codes

- optionally a null terminated string (in the selected character set) representing the enterprise ID in ASN.1 dotted notation. This enterprise ID will be sent with the TRAP. If the null string is passed, then the agent uses the sub-agent Identifier (OID as passed with the DPI OPEN packet) as the Enterprise ID.

o optionally a set of one or more name/type/length/value tuples. representing varBinds to be sent with the trap. Each varBind consists of:

- two null terminated strings (in the selected character set) representing the group and instance ID in ASN.1 dotted notation.

- the type, value length and value of the object that is returned.

The permitted types for the type field are defined in Table 17. See 3.3.4, "Value Representation" for information on how the value data is represented in the packet value field. 


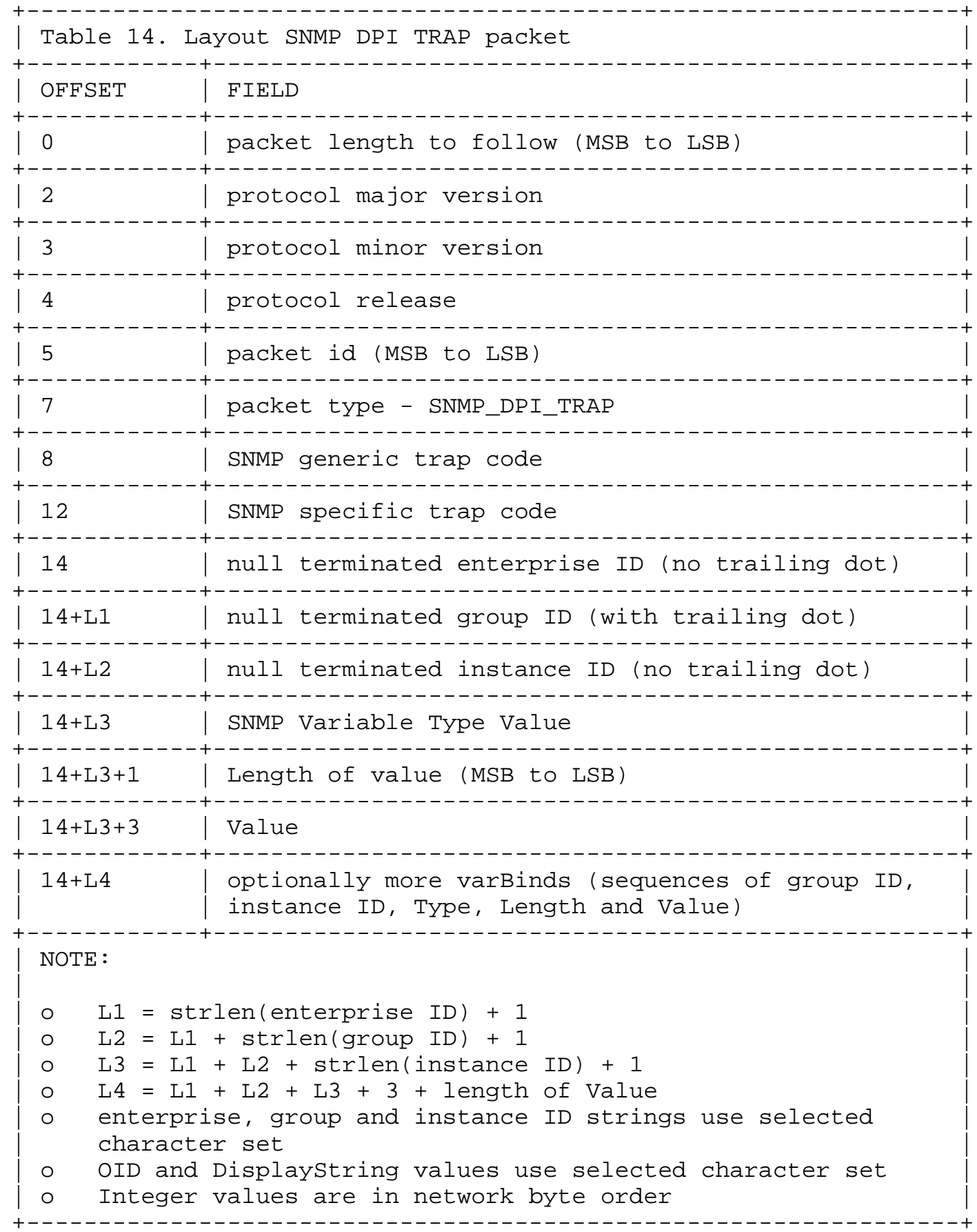




\subsection{CONSTANTS AND VALUES}

This section describes the constants that have been defined for this version of the SNMP DPI Protocol.

\subsubsection{PROTOCOL VERSION AND RELEASE VALUES}

\begin{tabular}{|c|c|}
\hline FIELD & VALUE \\
\hline protocol major version & 2 (SNMP DPI protocol) \\
\hline protocol minor version & 2 (version 2) \\
\hline protocol release & 0 (release 0$)$ \\
\hline
\end{tabular}

Previous versions of this protocol exist and should preferably be supported by an agent:

- version 1 , release 0 , described in [7]

Previous internal versions of this protocol exist and may or may not be supported by an agent:

- version 1, release 1, experimental within IBM.

o version 1, release 2, experimental within BNR. 


\subsubsection{PACKET TYPE VALUES}

\begin{tabular}{|c|c|}
\hline VALUE & PACKET TYPE \\
\hline 1 & SNMP_DPI_GET \\
\hline 2 & SNMP_DPI_GETNEXT \\
\hline 3 & SNMP_DPI_SET \\
\hline 4 & SNMP_DPI_TRAP \\
\hline 5 & SNMP_DPI_RESPONSE \\
\hline 6 & SNMP_DPI_REGISTER \\
\hline 7 & SNMP_DPI_UNREGISTER \\
\hline 8 & SNMP_DPI_OPEN \\
\hline 9 & SNMP_DPI_CLOSE \\
\hline 10 & SNMP_DPI_COMMIT \\
\hline 11 & SNMP_DPI_UNDO \\
\hline 12 & SNMP_DPI_GETBULK \\
\hline 13 & SNMP_DPI_TRAPV2 (not yet used) \\
\hline 14 & SNMP_DPI_INFORM (not yet used) \\
\hline 15 & SNMP_DPI_ARE_YOU_THERE \\
\hline
\end{tabular}




\subsubsection{VARIABLE TYPE VALUES}

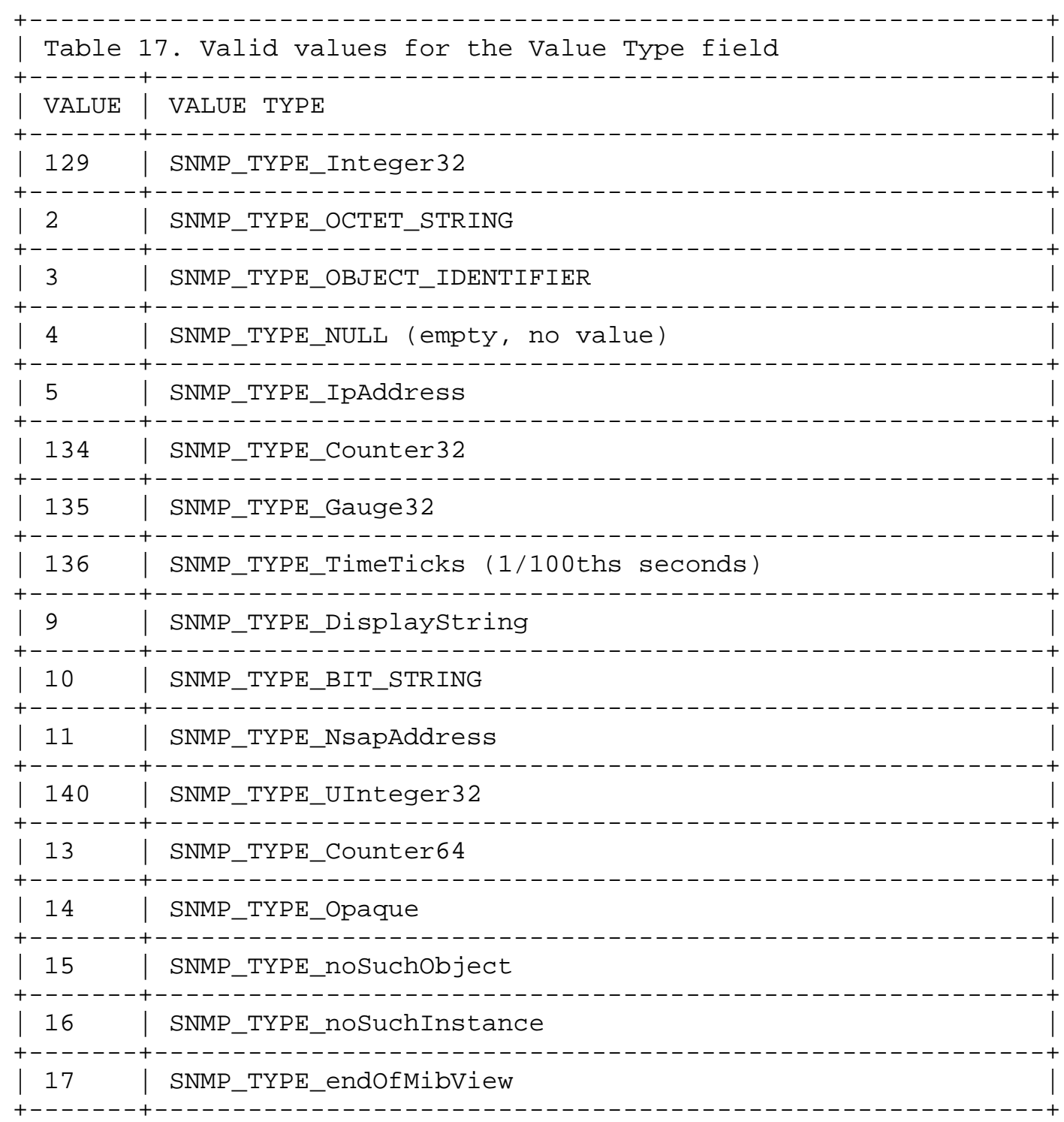

Notes:

1. A 32-bit integer value has its base base type ored with 128 .

2. Displaystring is a textual convention. An SNMP PDU shows a type of OCTET_STRING for the value. An agent can handle such an object as Displaystring if the object is included in some 
form of a compiled MIB for the agent. If not, the agent passes the value as an OCTET_STRING.

\subsubsection{VALUE REPRESENTATION}

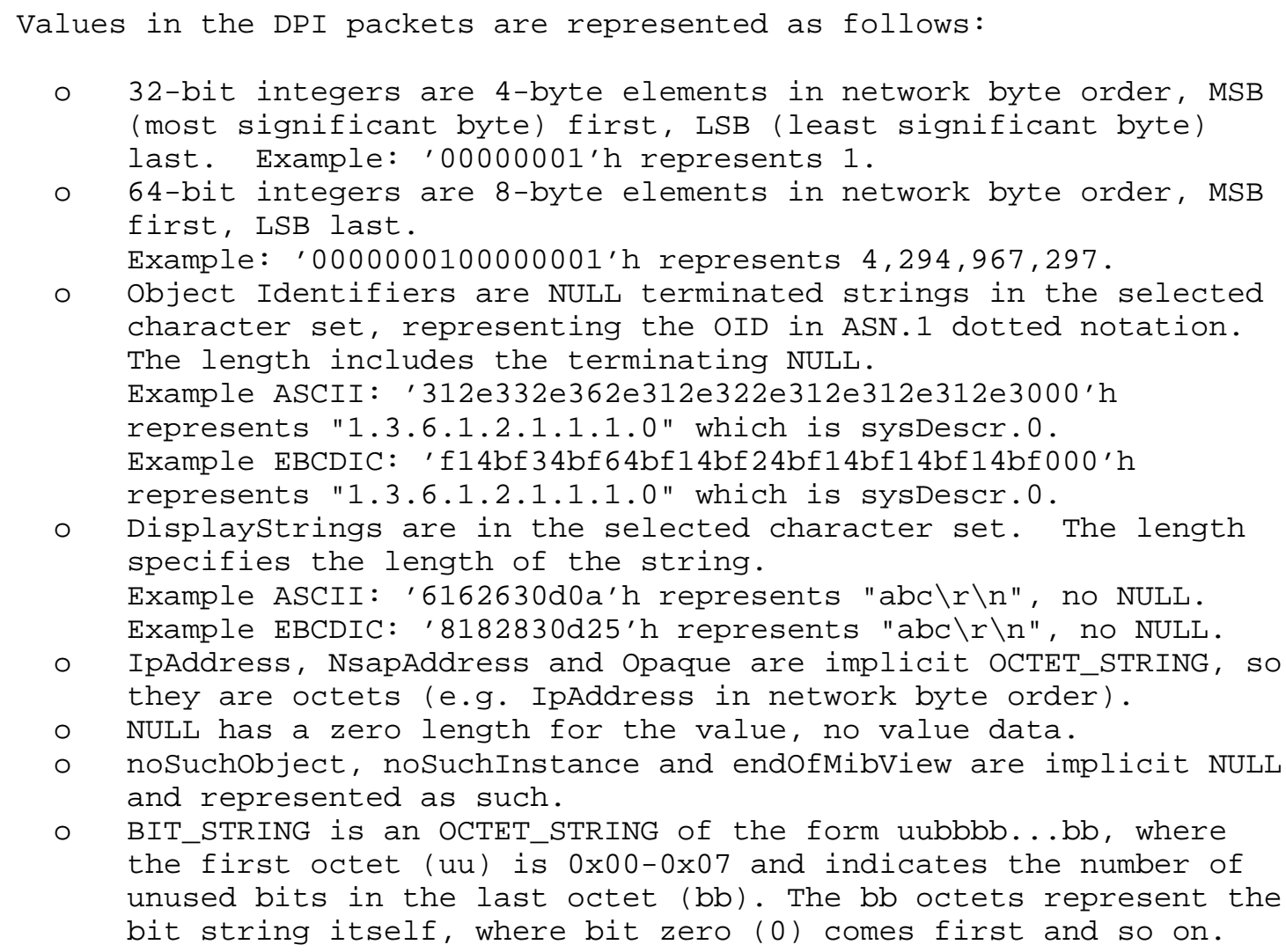

\subsubsection{CHARACTER SET SELECTION}

In the DPI OPEN packet, the sub-agent can specify the character set to be used for the representation of:

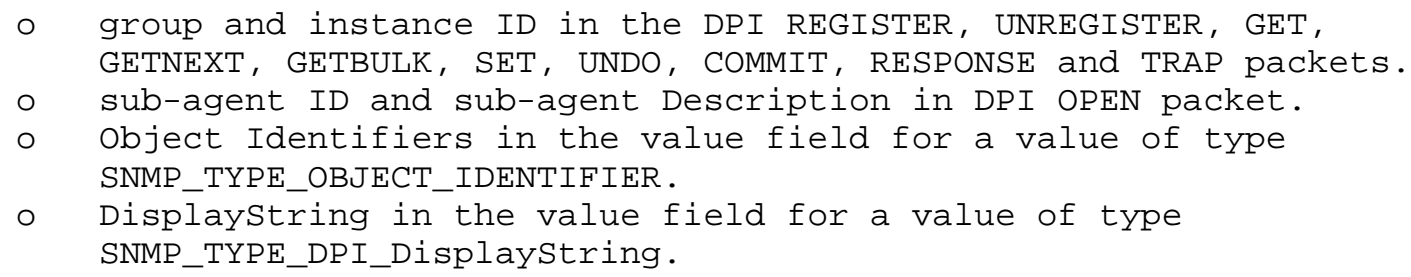

The choice is the native character set or the ASCII character set. 
The native set is the set native to the platform where the agent runs. If the native set is ASCII, then character set selection is a moot point. On non-ASCII based platforms, the agent must convert between native and ASCII if the native character set is chosen.

3.3.6 ERROR CODE VALUES FOR SNMP DPI RESPONSE PACKETS

When the RESPONSE packet is a response to a GET, GETNEXT, GETBULK, SET, COMMIT, or UNDO, then the error code can have one of the following values: 


\begin{tabular}{|c|c|}
\hline VALUE & ERROR CODE \\
\hline 0 & SNMP_ERROR_noError \\
\hline 1 & SNMP_ERROR_toOBig \\
\hline 2 & SNMP_ERROR_noSuchName (SNMPv1, do not use) \\
\hline 3 & SNMP_ERROR_badValue (SNMPv1, do not use) \\
\hline 4 & SNMP_ERROR_readOnly (SNMPv1 do not use) \\
\hline 5 & SNMP_ERROR_genErr \\
\hline 6 & SNMP_ERROR_noAccess \\
\hline 7 & SNMP_ERROR_wrongType \\
\hline 8 & SNMP_ERROR_wrongLength \\
\hline 9 & SNMP_ERROR_wrongEncoding \\
\hline 10 & SNMP_ERROR_wrongValue \\
\hline 11 & SNMP_ERROR_noCreation \\
\hline 12 & SNMP_ERROR_inconsistentValue \\
\hline 13 & SNMP_ERROR_resourceUnavailable \\
\hline 14 & SNMP_ERROR_commitFailed \\
\hline 15 & SNMP_ERROR_undoFailed \\
\hline 16 & SNMP_ERROR_authorizationError \\
\hline 17 & SNMP_ERROR_notWritable \\
\hline 18 & SNMP_ERROR_inconsistentName \\
\hline
\end{tabular}


When the RESPONSE packet is a response to an OPEN, REGISTER or UNREGISTER, then the error code can have one of the following values:

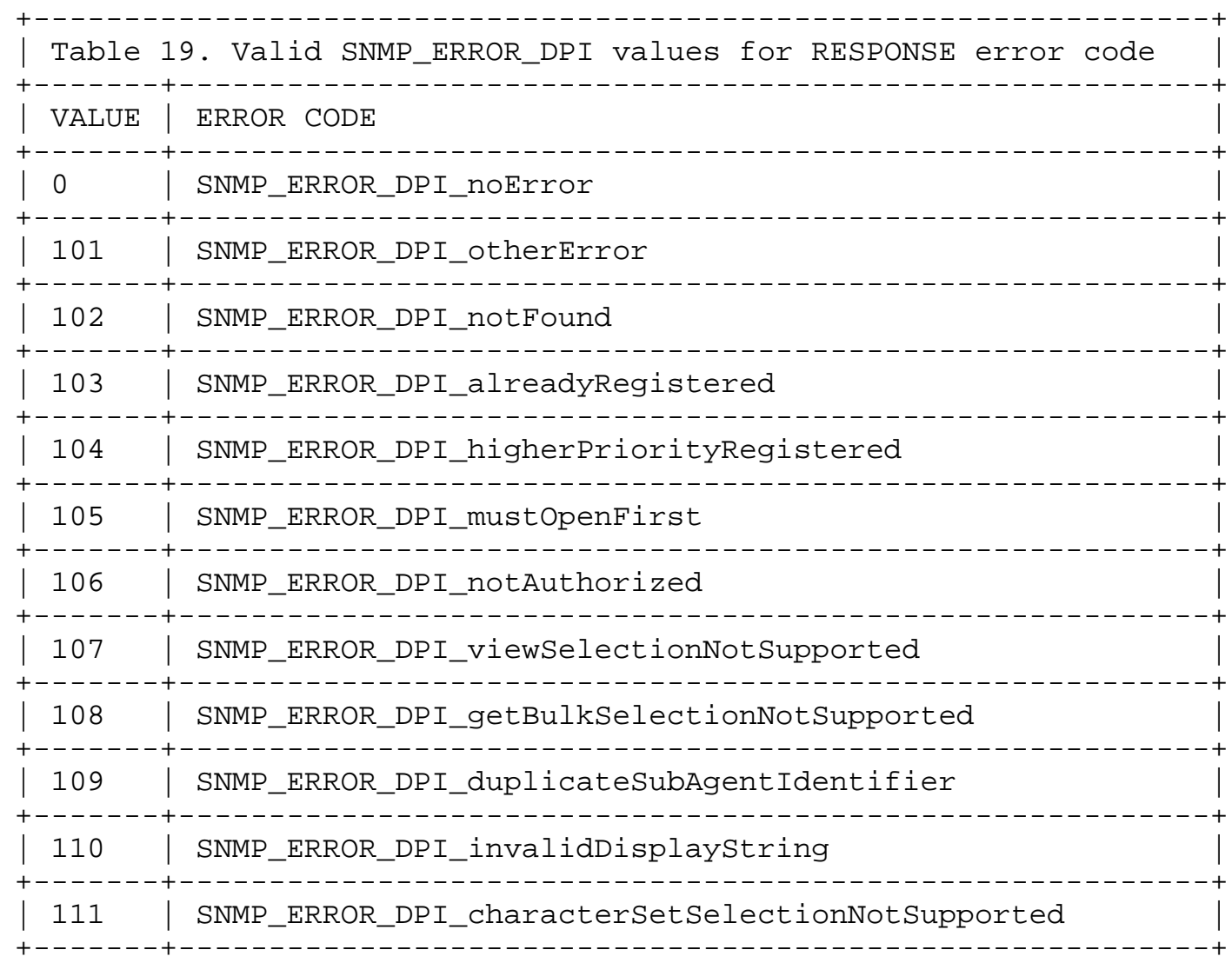




\subsubsection{UNREGISTER REASON CODES}

The following are valid reason codes in an UNREGISTER packet.

\begin{tabular}{|c|c|}
\hline VALUE & REASON CODE \\
\hline 1 & SNMP_UNREGISTER_otherReason \\
\hline 2 & SNMP_UNREGISTER_goingDown \\
\hline 3 & SNMP_UNREGISTER_justUnregister \\
\hline 4 & SNMP_UNREGISTER_newRegistration \\
\hline 5 & SNMP_UNREGISTER_higherPriorityRegistered \\
\hline 6 & SNMP_UNREGISTER_byManager \\
\hline 7 & SNMP_UNREGISTER_timeout \\
\hline
\end{tabular}




\subsubsection{CLOSE REASON CODES}

The following are valid reason codes in a CLOSE packet.

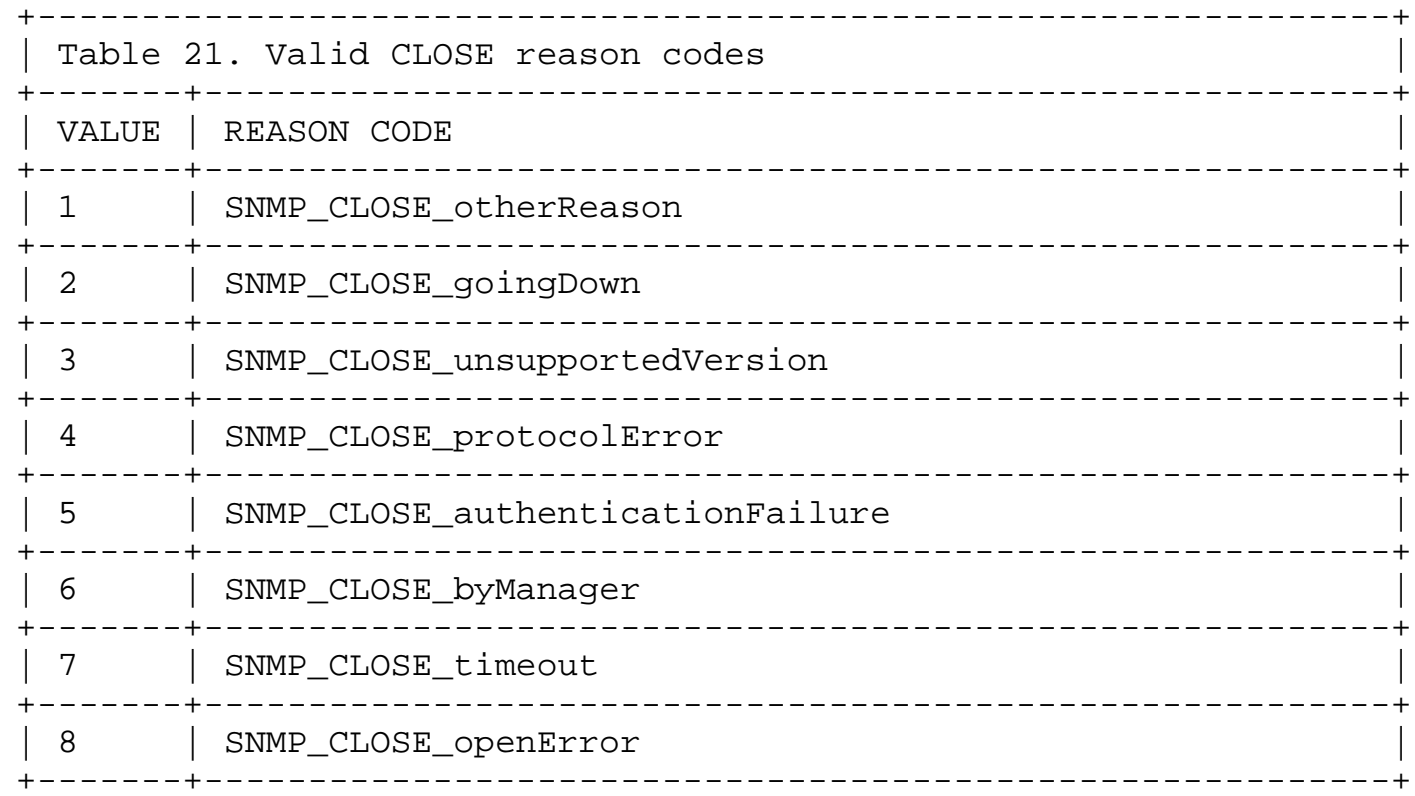

4. DPI 2.0 MIB DEFINITION

DPI20-MIB DEFINITIONS : := BEGIN

-- Objects in this MIB are implemented in the local SNMP agent. IMPORTS

MODULE-IDENTITY, OBJECT-TYPE, snmpModules, enterprises FROM SNMPV2-SMI

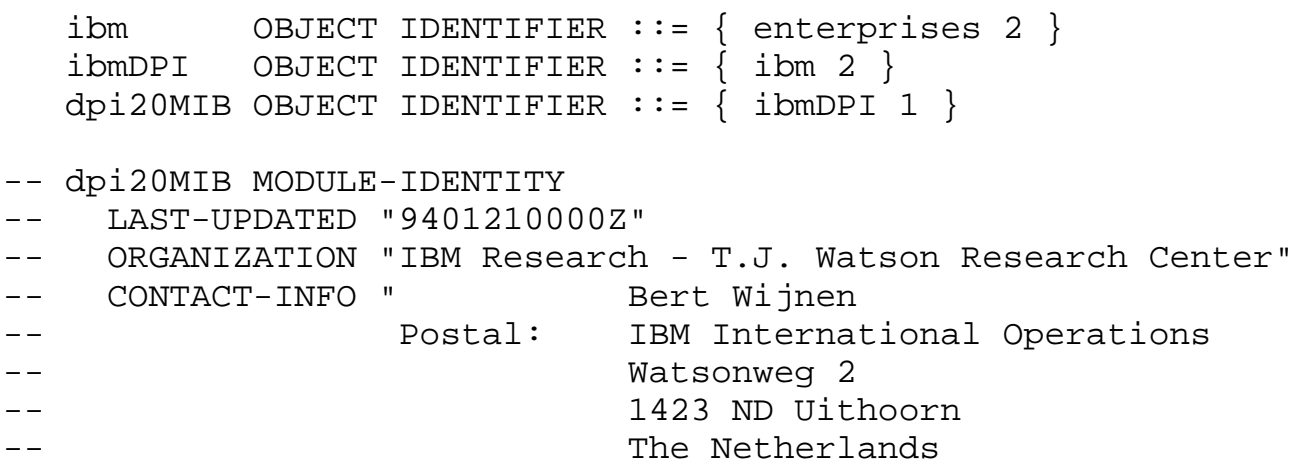

Wijnen, Carpenter, Curran, Sehgal \& Waters 


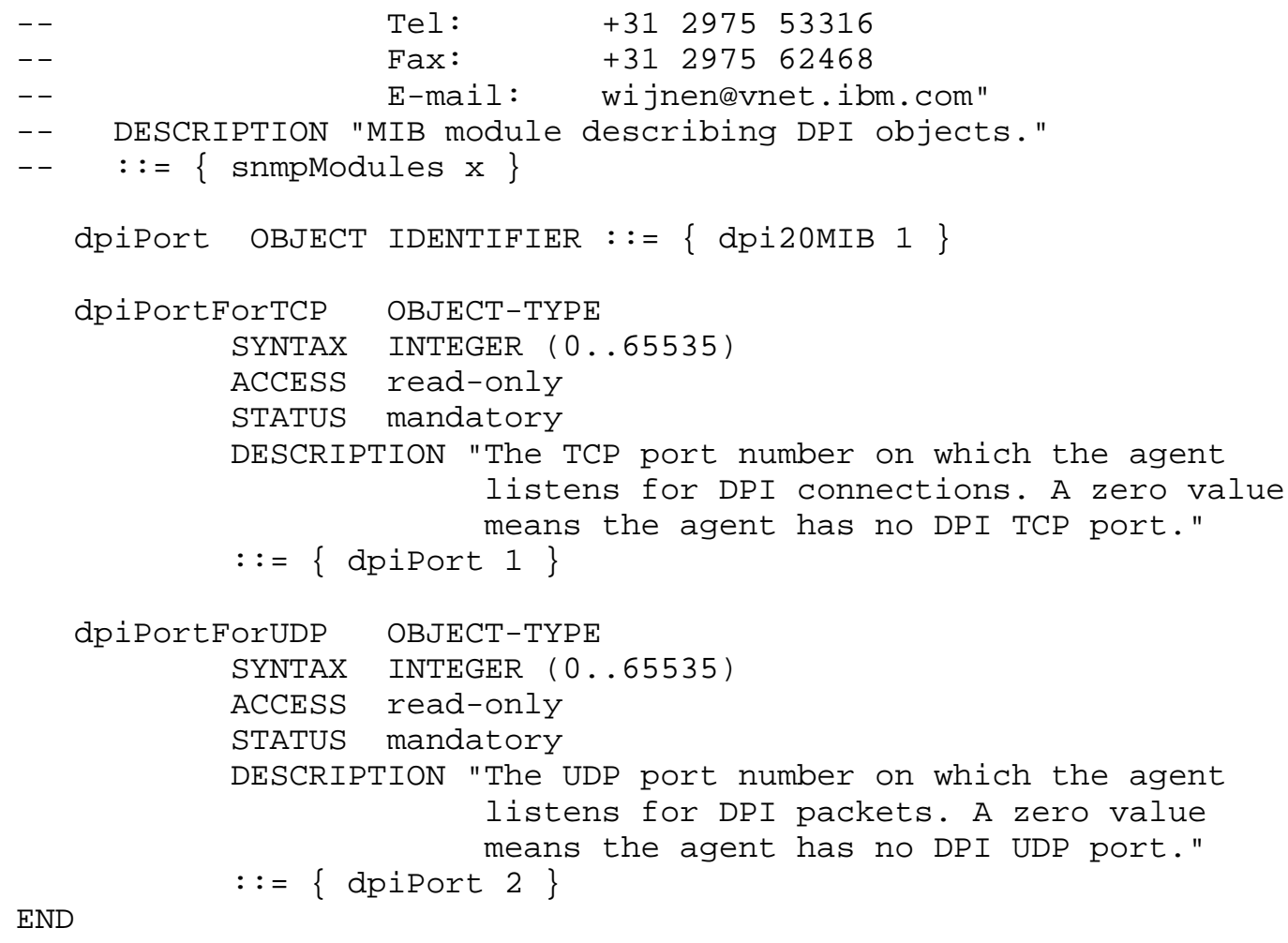

\section{SUBAGENT CONSIDERATIONS}

When implementing a sub-agent, it is strongly recommended to use the DPI version 2 approach (SNMPv2 based). This means:

- Use SNMPv2 error codes only (even though we have definitions for the old SNMPvi error codes).

o Do implement SET, COMMIT, UNDO processing properly.

- For GET requests, use the SNMPv2 approach and pass back noSuchInstance or noSuchobject value if such is the case. Continue to process all remaining varBinds in this case.

o For GETNEXT, use the SNMPV2 approach and pass back endOfMibView value if such is the case. Continue to process all remaining varBinds in this case.

- When you are processing a request from the agent (GET, GETNEXT, GETBULK, SET, COMMIT, UNDO) you are supposed to respond within the timeout period (which you can specify in the OPEN and REGISTER packets). If you fail to respond within that timeout period, the agent will most probably close your DPI connection and then discard your RESPONSE packet if it comes in later. If you can detect that the response is not going to make it in 
time, then you might decide to abort the request and return an SNMP_ERROR_genErr in the RESPONSE.

o If you have a UDP "connected" sub-agent, or one that uses another unreliable protocol, you may want to issue an SNMP DPI ARE_YOU_THERE request once in a while to ensure that the agent is still alive and still knows about you.

- When you are running on an EBCDIC based machine, and you use the (default) native character set, then all OID strings (as used for things like group ID, instance ID, Enterprise ID, sub-agent ID) and also all variable values of type OBJECT_IDENTIFIER or Displaystring will be passed to you in EBCDIC format. When you return a response, you should then also use EBCDIC FORMAT.

- When you are running on an EBCDIC based machine, and you use the ASCII character set (specified in DPI OPEN), then all OID strings (as used for things like group ID, instance ID, Enterprise ID, sub-agent ID) and also all variable values of type OBJECT_IDENTIFIER or Displaystring will be passed to you in ASCII format. When you return a response, you should then also use ASCII FORMAT.

- When you are running on an ASCII machine, then the character set selection for you basically is moot. Except maybe when you connect to an EBCDIC based agent, in which case you may want to specify in the DPI OPEN packet that you want to use ASCII character set. After that, all this is transparent to you and the burden of conversion is on the EBCDIC based agent.

- Please realize that Displaystring is only a textual convention. In the SNMP PDU (SNMP packet), the type is just an OCTET_STRING, and from that it is not clear if this is a Displaystring or any arbitrary data. This means that the agent can only know about an object being a Displaystring if the object is included in some sort of a compiled MIB. If it is, then the agent will use SNMP_TYPE_DisplayString in the type field of the varBind in a DPI SET packet. When you send a Displaystring in a RESPONSE packet, the agent will handle it as such (e.g. translate EBCDIC to ASCII if needed).

\subsection{DPI API}

The primary goal of this document is to specify the SNMP DPI, a protocol by which sub-agents can exchange SNMP related information with an agent. On top of this protocol, one can imagine one or possibly many Application Programming Interfaces, but those are not addressed in this document. 
However, in order to provide an environment that is more or less platform independent, we strongly suggest to also define a DPI API. We have a sample DPI API available, see 9, "Sample sources for Anonymous FTP" for a place to obtain that sample DPI API.

\subsection{OVERVIEW OF REQUEST PROCESSING}

\subsubsection{GET PROCESSING}

A GET request is the easiest to process. The DPI GET packet holds one or more varBinds that the sub-agent has taken responsibility for.

If the sub-agent encounters an error while processing the request, it creates a DPI RESPONSE packet with an appropriate error indication in the error_code field and sets the error_index to the position of the varBind at which the error occurs (first varBind is index 1, second varBind is index 2, and so on). No name/type/length/value information needs to be provided in the packet, because by definition, the varBind information is the same as in the request to which this is a response, and the agent still has that information.

If there are no errors, then the sub-agent creates a DPI RESPONSE packet in which the error_code is set to SNMP_ERROR_noError (zero) and error_index is set to zero. The packet must also include the name/type/length/value of each varBind requested. When you get a request for a non-existing object or a non-existing instance of an object, then you must return a NULL value with a type of SNMP_TYPE_noSuchobject or SNMP_TYPE_noSuchInstance respectively.

These two values are not considered errors, so the error_code and error_index should be zero.

The DPI RESPONSE packet is then sent back to the agent.

\subsubsection{SET PROCESSING}

Processing a DPI SET request is more difficult than a DPI GET request. In the case of a DPI SET packet, additional information is available in the packet, namely the value type, value length and value to be set.

If the sub-agent encounters an error while processing the request, it creates a DPI RESPONSE packet with an appropriate error indication in the error_code field and an error_index listing the position of the varBind at which the error occurs (first varBind is index 1, second varBind is index 2, and so on). No name/type/length/value information needs to provided in the packet, because by definition, the varBind information is the same as in the request to which this 
is a response, and the agent still has that information.

If there are no errors, then the sub-agent creates a DPI RESPONSE packet in which the error_code is set to SNMP_ERROR_noError (zero) and error_index is set to zero. No name/type/length/value information is needed; by definition the RESPONSE to a SET should contain exactly the same varBind data as the data present in the request, so the agent can use the values it already has. (This suggests that the agent must keep state information, and that is indeed the case. It needs to do that anyway in order to be able to later pass the data with a DPI COMMIT or DPI UNDO packet). The subagent must have allocated the required resources and prepared itself for the SET. It does not yet effectuate the set, that will be done at COMMIT time.

The sub-agent sends a DPI RESPONSE packet (indicating success or failure for the preparation phase) back to the agent.

The agent will then issue a SET request for all other varBinds in the same original SNMP request it received. This may be to the same or to one or more different sub-agents. Once all SET requests have returned a "no error" condition, the agent starts sending DPI COMMIT packets to the sub-agent(s). If any SET request returns an error, then the agent sends DPI UNDO packets to those sub-agents that indicated successful processing of the SET preparation phase.

When the sub-agent receives the DPI COMMIT packet, again all the varBind information will be available in the packet. The sub-agent can now effectuate the SET request.

If the sub-agent encounters an error while processing the COMMIT request, it creates a DPI RESPONSE packet with value SNMP_ERROR_commitFailed in the error_code field and an error_index that lists at which varBind the error occurs (first varBind is index 1 and so on). No name/type/length/value information is needed. The fact that a commitfailed error exists does not mean that this error should be returned easily. A sub-agent should do all that is possible to make a COMMIT succeed.

If there are no errors, and the SET/COMMIT has been effectuated with success, then the sub-agent creates a DPI RESPONSE packet in which the error_code is set to SNMP_ERROR_noError (zero) and error_index is set to zero. No name/type/length/value information is needed.

So far we have discussed a SET, COMMIT sequence. That happens if all goes well. However, after a successful SET, the sub-agent may receive a DPI UNDO packet. The sub-agent must now undo any preparations it made during the SET processing (like free allocated 
memory and such). Even after a COMMIT, a sub-agent may still receive a DPI UNDO packet. This is the case if some other sub-agent could not complete a COMMIT request. Because of the SNMP-requirement that all varBinds in a single SNMP SET request must be changed "as if simultaneous", all committed changes must be undone if any of the COMMIT requests fail. In this case the sub-agent must try and undo the committed SET operation.

If the sub-agent encounters an error while processing the UNDO request, it creates a DPI RESPONSE packet with value SNMP_ERROR_undoFailed in the error_code field and an error_index that lists at which varBind the error occurs (first varBind is index 1 and so on). No name/type/length/value information is needed. The fact that an undoFailed error exists does not mean that this error should be returned easily. A sub-agent should do all that is possible to make an UNDO succeed.

If there are no errors, and the UNDO has been effectuated with success, then the sub-agent creates a DPI RESPONSE packet in which the error_code is set to SNMP_ERROR_noError (zero) and error_index is set to zero. No name/type/length/value information is needed.

\subsubsection{GETNEXT PROCESSING}

GETNEXT requests are a bit more complicated to process than a GET. The DPI GETNEXT packet contains the object(s) on which the GETNEXT operation must be performed. The semantics of the operation are that the sub-agent is to return the name/type/length/value of the next variable it supports whose (ASN.1) name lexicographically follows the one passed in the group ID (sub-tree) and instance ID.

In this case, the instance ID may not be present (NULL) implying that the NEXT object must be the first instance of the first object in the sub-tree that was registered.

It is important to realize that a given sub-agent may support several discontiguous sections of the MIB tree. In such a situation it would be incorrect to jump from one section to another. This problem is correctly handled by examining the group ID in the DPI packet. This group ID represents the "reason" why the sub-agent is being called. It holds the prefix of the tree that the sub-agent had indicated it supported (registered).

If the next variable supported by the sub-agent does not begin with that prefix, the sub-agent must return the same object instance as in the request (e.g. group ID and instance ID) with a value of SNMP_TYPE_endOfMibView (implied NULL value). This endOfMibView is not considered an error, so the error_code and error_index should be 
zero. If required, the SNMP agent will call upon the sub-agent again, but pass it a different group ID (prefix). This is illustrated in the discussion below.

Assume there are two sub-agents. The first sub-agent registers two distinct sections of the tree, $A$ and $C$. In reality, the sub-agent supports variables A.1 and A.2, but it correctly registers the minimal prefix required to uniquely identify the variable class it supports.

The second sub-agent registers a different section, B, which appears between the two sections registered by the first agent.

If a management station begins dumping the MIB, starting from $A$, the following sequence of queries of the form get-next(group ID, instance ID) would be performed:

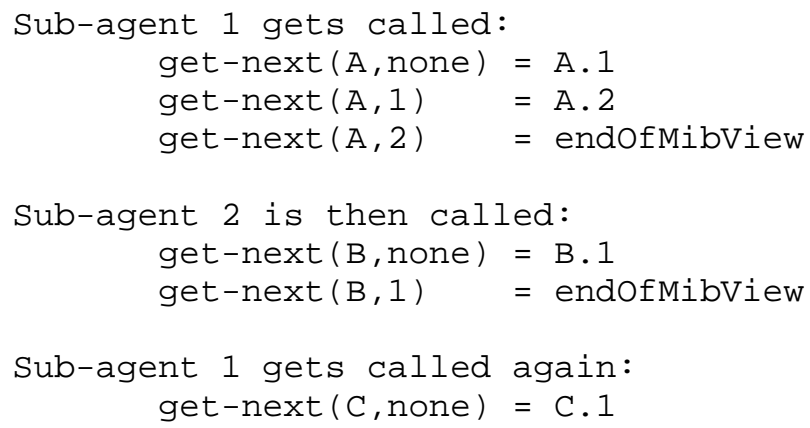

\subsubsection{GETBULK PROCESSING}

You can ask the agent to translate GETBULK requests into multiple GETNEXT requests. This is basically the default and it is specified in the DPI REGISTER packet. In principle, we expect the majority of DPI sub-agents to run on the same machine as the agent (or otherwise, on the same physical network), so repetitive GETNEXT requests stay local and in general should not be a problem.

If experience tells us different, the sub-agent can tell the agent to pass on a DPI GETBULK packet.

When a GETBULK request is received, the sub-agent must process the request and send a RESPONSE that sends back as many varBinds as requested by the request, as long as they fit with in the buffers.

The GETBULK requires similar processing as a GETNEXT with regard to endOfMibView handling. 


\subsubsection{OPEN REQUEST}

As the very first step, a DPI sub-agent must open a "connection" with the agent. To do so, it must send a DPI OPEN packet in which these things must be specified:

- The max timeout value in seconds. The agent is requested to wait this long for a response to any request for an object being handled by this sub-agent. The agent may have an absolute maximum timeout value which will be used if the sub-agent asks for too big a timeout value. A value of zero can be used to indicate that the agent's own default timeout value should be used. A sub-agent is advised to use a reasonably short interval of a few seconds or so. If a specific sub-tree needs a (much) longer time, then a specific REGISTER can be done for that sub-tree with a longer timeout value.

- The maximum number of varBinds that the sub-agent is prepared to handle per DPI packet. Specifying 1 would result in DPI version 1 behavior of one varBind per DPI packet that the agent sends to the sub-agent.

- The character set you want to use. By default (value 0) this is the native character set of the machine (platform) where the agent runs.

Since the sub-agent and agent normally run on the same system or platform, you want to use the native character set (which on many platforms is ASCII anyway).

If your platform is EBCDIC based, then using the native character set of EBCDIC makes it easy to recognize the string representations of the fields like group ID, instance ID, etc.

At the same time, the agent will translate the value from ASCII NVT to EBCDIC (and vice versa) for objects that it knows (from a compiled MIB) to have a textual convention of Displaystring. Be aware that this fact cannot be determined from the SNMP PDU encoding because in the PDU the object is only known to be an OCTET_STRING.

If your sub-agent runs on an ASCII based platform and the agent runs on an EBCDIC based platform (or the other way around), then you can specify that you want to use the ASCII character set, and so you both know how to handle the string-based data. Beware that not all agents need to support other than native character set selection. See 5, "Subagent Considerations" and 3.3.5, "Character set selection" for more information on character set usage.

- The sub-agent ID. This an ASN.1 Object Identifier that uniquely identifies the sub-agent. This OID is represented as a null terminated string using the selected character set. 
Example: "1.3.5.1.2.3.4.5".

- The sub-agent Description. This is a Displaystring describing the sub-agent. This is a character string using the selected character set. Example: "DPI sample sub-agent version 2.0"

Once a sub-agent has sent a DPI OPEN packet to an agent, it should expect a DPI RESPONSE packet that informs the sub-agent about the result of the request. The packet ID of the RESPONSE packet should be the same as that of the OPEN request to which the RESPONSE packet is the response. See Table 19 for a list of valid DPI RESPONSE error codes that may be expected. If you receive an error RESPONSE on the OPEN packet, then you will also receive a DPI CLOSE packet with an SNMP_CLOSE_openError code, and then the agent closes the "connection".

If the OPEN is accepted, then the next step is to REGISTER one or more MIB sub-trees.

\subsubsection{CLOSE REQUEST}

When a sub-agent is finished and wants to terminate it should first UNREGISTER its sub-trees and then close the "connection" with the agent. To do so, it must send a DPI CLOSE packet in which it specifies a reason for the closing. See Table 21 for a list of valid CLOSE reason codes. You should not expect a response to the CLOSE request.

A sub-agent should also be prepared to handle an incoming DPI CLOSE packet from the agent. Again, the packet will contain a reason code for the CLOSE request. A sub-agent need not send a response to a CLOSE request. The agent just assumes that the sub-agent will handle it appropriately. The close takes place, no matter what the subagent does with it.

\subsubsection{REGISTER REQUEST}

Before a sub-agent will receive any requests for MIB variables it must first register the variables or sub-tree it supports with the SNMP agent. The sub-agent must specify a number of things in the REGISTER request:

o The sub-tree to be registered. This is a null terminated string in the selected character set. The sub-tree must have a trailing dot (example: "1.3.6.1.2.3.4.5.").

o The requested priority for the registration, one of: -1 Request for best available priority.

0 Request for next better available priority than highest priority currently registered for this sub-tree. 
NNN Any other positive value requests that specific priority if available or the first worse priority that is available.

o The max timeout value in seconds. The agent is requested to wait this long for a response to any request for an object in this sub-tree. The agent may have an absolute maximum timeout value which will be used if the sub-agents asks for too big a timeout value. A value of zero can be used to indicate that the DPI OPEN value should be used for timeout.

- A specification if the sub-agent wants to do view selection. If it does, then the community name (from SNMPv1 packets) will be passed in the DPI GET, GETNEXT, SET packets).

- A specification if the sub-agent wants to receive GETBULK packets or if it just prefers that the agent converts a GETBULK into multiple GETNEXT requests.

Once a sub-agent has sent a DPI REGISTER packet to the agent, it should expect a DPI RESPONSE packet that informs the sub-agent about the result of the request. The packet ID of the RESPONSE packet should be the same as that of the REGISTER packet to which the RESPONSE packet is the response. If the response indicates success, then the error_index field in the RESPONSE packet contains the priority that the agent assigned to the sub-tree registration. See Table 19 for a list of valid DPI RESPONSE error codes that may be expected.

\subsubsection{UNREGISTER REQUEST}

A sub-agent may unregister a previously registered sub-tree. The sub-agent must specify a few things in the UNREGISTER request:

o The sub-tree to be unregistered. This is a null terminated string in the selected character set. The sub-tree must have a trailing dot (example: "1.3.6.1.2.3.4.5.").

o The reason for the unregister. See Table 20 for a list of valid reason codes.

Once a sub-agent has sent a DPI UNREGISTER packet to the agent, it should expect a DPI RESPONSE packet that informs the sub-agent about the result of the request. The packet ID of the RESPONSE packet should be the same as that of the REGISTER packet to which the RESPONSE packet is the response. See Table 19 for a list of valid DPI RESPONSE error codes that may be expected.

A sub-agent should also be prepared to handle incoming DPI UNREGISTER packets from the agent. Again, the DPI packet will contain a reason code for the UNREGISTER. A sub-agent need not send a response to an UNREGISTER request. The agent just assumes that the sub-agent will handle it appropriately. The registration is removed, no matter what 
the sub-agent returns.

\subsubsection{TRAP REQUEST}

A sub-agent can request that the SNMP agent generates a trap for it. The sub-agent must provide the desired values for the generic and specific parameters of the trap. It may optionally provide a set of one or more name/type/length/value tuples that will be included in the trap packet. Also, it may optionally specify an Enterprise ID (Object Identifier) for the trap to be generated. If a NULL value is specified for the Enterprise ID, then the agent will use the subagent Identifier (from the DPI OPEN packet) as the Enterprise ID to be sent with the trap.

\subsubsection{ARE_YOU_THERE REQUEST}

A sub-agent can send an ARE_YOU_THERE packet to the agent. This may be useful to do if you have a DPI "connection" over an unreliable transport protocol (like UDP).

If the "connection" is in a healthy state, the agent responds with a RESPONSE packet with SNMP_ERROR_DPI_noError.

If the "connection" is not in a healthy state, the agent may respond with a RESPONSE packet with an error indication, but the agent might not react at all, so you would timeout while waiting for a response.

\subsubsection{HOW TO QUERY THE DPI PORT.}

The DPI API implementations are encouraged to provide a facility that helps DPI sub-agent programmers to dynamically find the port that the agent is using for the TCP and/or UDP DPI port(s). A suggested name for such a function is: query_DPI_port().

\section{REFERENCES}

[1] Case, J., Fedor, M., Schoffstall M., and J. Davin, "Simple Network Management Protocol (SNMP) ", STD 15, RFC 1157, SNMP Research, Performance Systems International, MIT Laboratory for Computer Science, May 1990.

[2] Information processing systems - Open Systems Interconnection, "Specification of Abstract Syntax Notation One (ASN.1)", International Organization for Standardization, International Standard 8824, December 1987. 
[3] Information processing systems - Open Systems Interconnection, "Specification of Basic Encoding Rules for Abstract Syntax Notation One (ASN.1)", International Organization for Standardization, International Standard 8825, December 1987.

[4] McCloghrie, K., and M. Rose, "Management Information Base for Network Management of TCP/IP-based internets: MIB II", STD 17, RFC 1213, Hughes LAN Systems, Performance Systems International, March 1991.

[5] Rose, M., and K. McCloghrie, "Structure and Identification of Management Information for TCP/IP-based internets", STD 16, RFC 1155, Performance Systems International, Hughes LAN Systems, May 1990 .

[6] Rose, M., "SNMP MUX Protocol and MIB", RFC 1227, Performance Systems International, RFC 1227, May 1991.

[7] Carpenter G., and B. Wijnen, "SNMP-DPI, Simple Network Management Protocol Distributed Program Interface", RFC 1228, International Business Machines, Inc., May 1991.

[8] Case, J., McCloghrie, K., Rose, M., and S. Waldbusser, "SNMPv2 RFCs (RFC 1441 through RFC 1452)", SNMP Research Inc, Hughes LAN Systems, Dover Beach Consulting Inc, Carnegie Mellon University, Trusted Information Systems, April 1993.

[9] International Business Machines, Inc., TCP/IP for VM: Programmer's Reference, SC31-6084-0, 1990.

[10] International Business Machines, Inc., Virtual Machine System Facilities for Programming, Release 6, SC24-5288-01, 1988.

\section{SECURITY CONSIDERATIONS}

Security issues are not discussed in this memo. 
8. AUTHORS' ADDRESSES

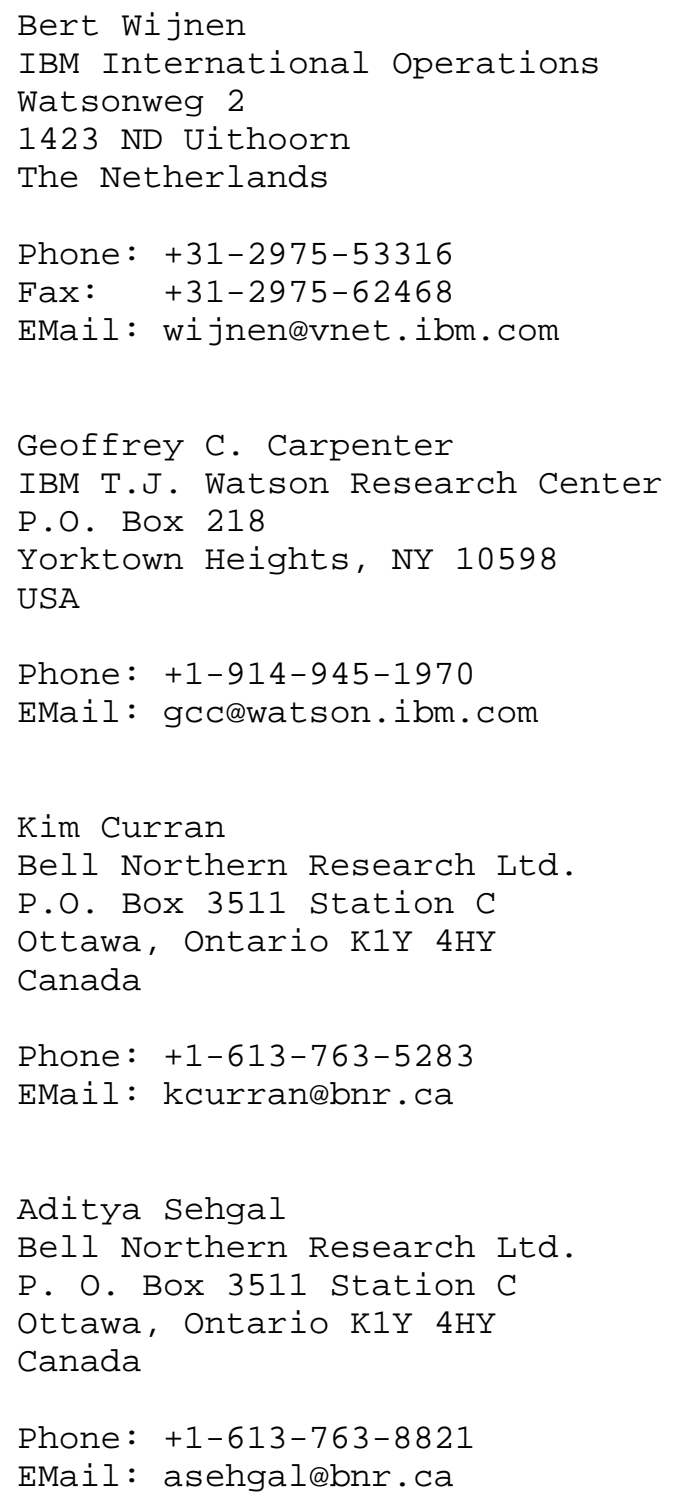


Glen Waters

Bell Northern Research Ltd.

P.O. Box 3511 Station C

Ottawa, Ontario K1Y $4 \mathrm{HY}$

Canada

Phone: +1-613-763-3933

EMail: gwaters@bnr.ca

9. SAMPLE SOURCES FOR ANONYMOUS FTP

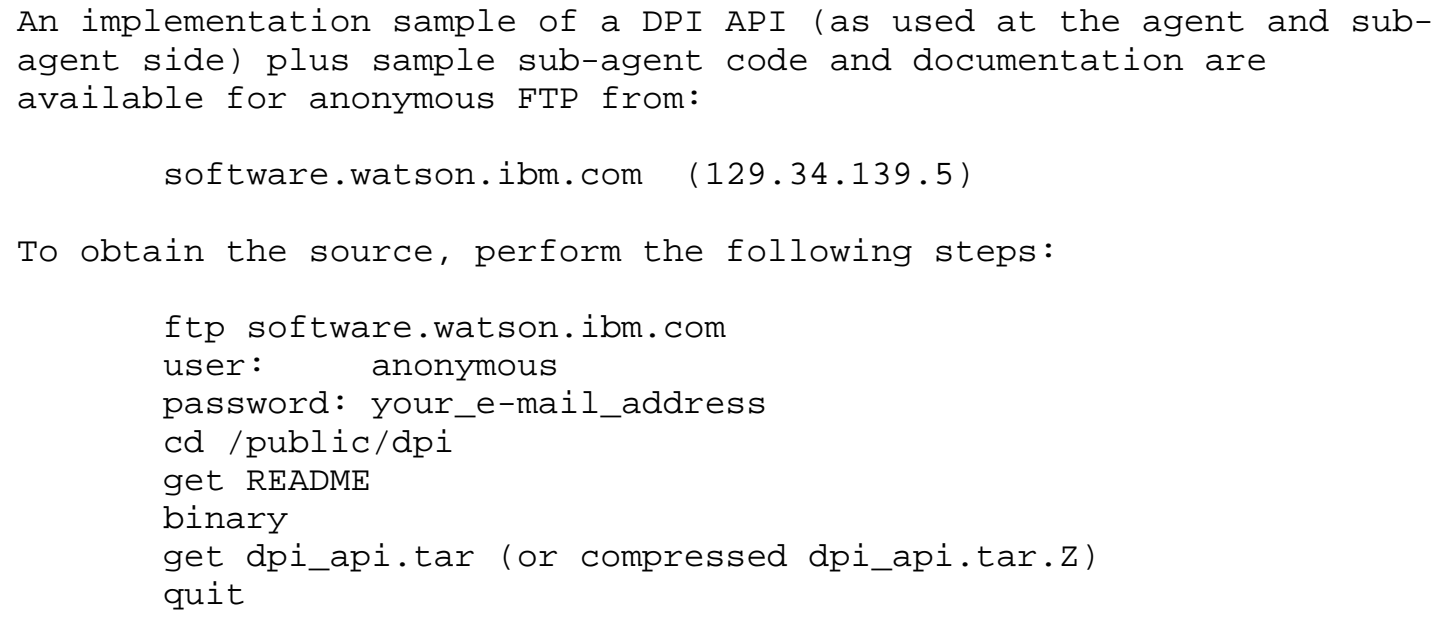

